\title{
Use of whole genome sequencing to determine genetic basis of suspected mitochondrial disorders: cohort study
}

\author{
Katherine R Schon, ${ }^{1,2,3}$ Rita Horvath, ${ }^{1}$ Wei Wei, ${ }^{1,2}$ Claudia Calabrese, ${ }^{1,2}$ Arianna Tucci, ${ }^{4}$ \\ Kristina Ibañez, ${ }^{4}$ Thiloka Ratnaike, ${ }^{1,2,5}$ Robert D S Pitceathly, ${ }^{6}$ Enrico Bugiardini, ${ }^{6}$ \\ Rosaline Quinlivan, ${ }^{6}$ Michael G Hanna, ${ }^{6}$ Emma Clement, ${ }^{7}$ Emma Ashton, ${ }^{8}$ John A Sayer, ${ }^{9}$ \\ Paul Brennan, ${ }^{10}$ Dragana Josifova, ${ }^{11}$ Louise Izatt, ${ }^{11}$ Carl Fratter, ${ }^{12}$ Victoria Nesbitt, ${ }^{12}$ \\ Timothy Barrett, ${ }^{13,14}$ Dominic J McMullen, ${ }^{13}$ Audrey Smith, ${ }^{15}$ Charulata Deshpande, ${ }^{11,15}$ \\ Sarah F Smithson, ${ }^{16}$ Richard Festenstein, ${ }^{17}$ Natalie Canham, ${ }^{18}$ Mark Caulfield, ${ }^{19}$ \\ Henry Houlden, ${ }^{6,20}$ Shamima Rahman, ${ }^{20,21,22}$ Patrick F Chinnery, ${ }^{1,2,20}$ on behalf of \\ the Genomics England Research Consortium
}

For numbered affiliations see end of the article

Correspondence to: $\mathrm{P}$ Chinnery pfc25@cam.ac.uk

(or @cam_mito on Twitte ORCID 0000-0002-7065-6617) Additional material is published online only. To view please visit the journal online.

Cite this as: $B M J$ 2021;375:e066288 http://dx.doi.org/10.1136/ bmj-2021-066288

Accepted: 11 October 2021

\section{ABSTRACT}

OBJECTIVE

To determine whether whole genome sequencing can be used to define the molecular basis of suspected mitochondrial disease.

\section{DESIGN}

Cohort study.

SETTING

National Health Service, England, including secondary and tertiary care.

\section{PARTICIPANTS}

345 patients with suspected mitochondrial disorders recruited to the 100000 Genomes Project in England between 2015 and 2018.

\section{INTERVENTION}

Short read whole genome sequencing was performed. Nuclear variants were prioritised on the basis of gene panels chosen according to phenotypes, ClinVar pathogenic/likely pathogenic variants, and the top 10 prioritised variants from Exomiser. Mitochondrial DNA variants were called using an in-house pipeline and compared with a list of pathogenic variants. Copy number variants and short tandem repeats for 13 neurological disorders were also analysed. American College of Medical Genetics guidelines were followed for classification of variants.

\section{WHAT IS ALREADY KNOW ON THIS TOPIC}

Mitochondrial disorders are among the most common inherited diseases, but a genetic diagnosis is not possible in around $40 \%$ of patients, limiting genetic counselling and prevention

Whole genome sequencing (WGS) has the potential to shorten the "diagnostic odyssey" for patients with suspected mitochondrial disorders

Use of WGS in a national healthcare system has not been previously investigated

\section{WHAT THIS STUDY ADDS}

After exclusion of common genetic causes, WGS identified a definite or probable genetic diagnosis in an additional $31 \%$ of patients, and another $2 \%$ had possible diagnoses

$62.5 \%$ of the families with a new diagnosis had a non-mitochondrial disorder, showing that a wide genomic approach is more useful than a targeted panel

testing approach

The new diagnoses included treatable disorders

\section{MAIN OUTCOME MEASURE}

Definite or probable genetic diagnosis.

\section{RESULTS}

A definite or probable genetic diagnosis was identified in $98 / 319$ (31\%) families, with an additional 6 (2\%) possible diagnoses. Fourteen of the diagnoses $(4 \%$ of the 319 families) explained only part of the clinical features. A total of 95 different genes were implicated. Of 104 families given a diagnosis, 39 (38\%) had a mitochondrial diagnosis and 65 (63\%) had a nonmitochondrial diagnosis.

\section{CONCLUSION}

Whole genome sequencing is a useful diagnostic test in patients with suspected mitochondrial disorders, yielding a diagnosis in a further $31 \%$ after exclusion of common causes. Most diagnoses were nonmitochondrial disorders and included developmental disorders with intellectual disability, epileptic encephalopathies, other metabolic disorders, cardiomyopathies, and leukodystrophies. These would have been missed if a targeted approach was taken, and some have specific treatments.

\section{Introduction}

Mitochondrial disorders haveemerged asa commoncause of inherited metabolic disease, affecting approximately 1 in 5000 people. ${ }^{1}$ They are caused by mutations in genes that primarily affect oxidative phosphorylation and ATP synthesis. ${ }^{2}$ Mitochondria are intracellular organelles that play a pivotal role in cellular energy metabolism. This is achieved by a series of complex enzymes located in the inner mitochondrial membrane that perform oxidative phosphorylation and synthesise ATP. ATP is a chemical source of energy needed for all active cellular processes. The impairment of mitochondrial function tends to affect tissues with high energy demand such as the brain, the peripheral nerves, the eye, the heart, and the peripheral muscles. Clinical diagnosis of mitochondrial disorders is challenging because they can affect a single organ, such as the eye in Leber hereditary optic neuropathy, ${ }^{3}$ or many different systems and can present at any age. Although some patients present with a classic mitochondrial syndrome, such as mitochondrial encephalomyopathy with lactic acidosis and stroke-like episodes, many present with only one or a few of the clinical features (oligosymptomatic cases) $^{4}$ or with an ill defined multisystem disorder. 
Mitochondria contain their own DNA in the form of a small $16.5 \mathrm{~kb}$ circle of double stranded DNA (mtDNA), which encodes for 13 peptides, two ribosomal RNAs, and 22 transfer RNAs that are essential for synthesising proteins within the organelle. However, the vast majority of proteins making up the mitochondria are encoded by genes in the nucleus and are synthesised in the cytosol before being imported through bespoke import machinery. Mitochondrial disorders can be caused by pathogenic variants in either the mtDNA or the nuclear genome, can follow any inheritance pattern (autosomal dominant, autosomal recessive, $\mathrm{X}$ linked, de novo, or maternal), and are highly genetically heterogeneous. ${ }^{5}$ For example, Leigh syndrome, the most common childhood presentation of mitochondrial disease, which usually presents in the first year of life with stepwise loss of skills, is caused by mutations in around 100 nuclear and mtDNA genes. ${ }^{6}$ These challenges often result in a prolonged patient journey from symptom onset to reaching a diagnosis, referred to as a "diagnostic odyssey." ${ }^{78}$ In patients with rare diseases, this typically involves multiple appointments-first in primary care and then with different specialist services-and many investigations, sometimes over many years. One survey found that patients saw an average of eight physicians before having a mitochondrial disease diagnosed, and $70 \%$ had a muscle biopsy. ${ }^{8}$

Diagnosis of mitochondrial disease has traditionally relied on an invasive tissue biopsy for biochemical and histochemical analysis, ${ }^{9}$ which can be normal even in patients with a defined genetic diagnosis. Sequencing a pre-defined list of genes known to cause a specific disorder (multi-gene panels) and sequencing of the protein coding regions (exons) of all genes (exome sequencing) have been effective for diagnosing mitochondrial disorders and in discovering new mitochondrial disease genes. ${ }^{10-16}$ However, making a genetic diagnosis is still not possible in about $40 \%$ of cases, ${ }^{10}$ even in highly selected cohorts-hence the need for new approaches.

A definitive genetic diagnosis benefits patients and families, ${ }^{17}$ allowing tailored information about prognosis and treatment, genetic counselling, and access to reproductive options such as prenatal diagnosis (genetic testing during pregnancy, usually by chorionic villus sampling or amniocentesis), preimplantation genetic diagnosis (the use of assisted reproductive technology and genetic testing of embryos), and mitochondrial transfer (replacing the mother's mitochondria in an ovum or early embryo with healthy mitochondria from another woman's donor egg or embryo, used for disorders caused by mtDNA mutations). ${ }^{18}$ Whole genome sequencing is a next generation sequencing technology that is used to sequence the entire genome of an individual. It has the added benefit of being able to diagnose pathogenic mutations affecting the mtDNA and the nuclear genome, ${ }^{19}$ so it has the potential to make a diagnosis in more families and shorten the time to diagnosis. ${ }^{20}$

The objective of this study was to determine whether whole genome sequencing could be used to define the molecular basis of suspected mitochondrial disorders in a national healthcare system in patients assessed in mainstream secondary care as well as tertiary centres. The 100000 Genomes Project was set up to introduce and embed genomic testing into the mainstream National Health Service (NHS), discover new disease genes, and make genetic diagnosis available for more patients. ${ }^{21}$ Following an initial pilot phase, ${ }^{22}$ patients in the 100000 Genomes Project were recruited from NHS genomic medicine centres across England. Here, we report the results for 345 patients with a suspected mitochondrial disorder recruited into the main programme between 2015 and 2018.

\section{Methods \\ Participants}

Participants with suspected mitochondrial disorders were recruited to the 100000 Genomes Project (main programme) between 2015 and 2018 with an unexplained multisystem progressive disorder usually involving the central nervous system, the neuromuscular system, or both. All participants provided written informed consent. Eligibility criteria stated that mtDNA and common nuclear genetic causes (eg, POLG mutations) should have been excluded (see supplementary methods for full inclusion criteria). All participants recruited under the category "suspected mitochondrial disorder" who had tiering data available in data release v8_2019-11-28 were included in the study. In 2015-18, genetic testing in the UK was arranged through 20 regional genetics laboratories, and there were three NHS highly specialised services for rare mitochondrial disorders. Testing of $P O L G$ and common mtDNA mutations (m.3243A $>\mathrm{G}$ associated with mitochondrial encephalomyopathy with lactic acidosis and stroke-like episodes and maternally inherited diabetes and deafness, m.8344A $>\mathrm{G}$ associated with myoclonic epilepsy with ragged red fibres, and m.8993T >G/C associated with Leigh syndrome and neurogenic muscle weakness ataxia and retinitis pigmentosa) in DNA extracted from blood was available through the highly specialised service laboratories. Further testing (such as gene panel testing) was available after discussion with the highly specialised services depending on the patient's clinical features (supplementary table A).

\section{Clinical information}

The referring clinician provided clinical information against a standardised list of clinical features as yes/ no answers (supplementary table B) or by using a standardised vocabulary for describing clinical features encountered in human disease (the Human Phenotype Ontology (HPO)). ${ }^{23}$ HPO classifies clinical features by organ system by using a branching tree incorporating increasing levels of detail. We used modified HPO terms to score participants using the Nijmegen Mitochondrial Disease Criteria ${ }^{24}{ }^{25}$ (supplementary table C), which use clinical features (muscular, central nervous system, and multisystem), magnetic resonance imaging and biochemical features, and muscle 
biopsy results to give a score out of 12 . We classified patients with a total score of $0-1$ as being unlikely to have a mitochondrial disorder, those with score 2-4 as a possible mitochondrial disorder, those with score 5-7 as a probable mitochondrial disorder, and those with score 8 or more as definite mitochondrial disorder. One investigator scored all the participants. A second investigator also scored 100 participants independently to develop the modifications for using the Nijmegen Mitochondrial Disease Criteria with HPO terms. For each HPO term, the HPO system was determined by following the branching tree back to the subtype of "phenotypic abnormality."

\section{Whole genome sequencing}

We extracted DNA from peripheral blood and quantified and sequenced it according to a national specification (Illumina TruSeq, HiSeq 2500, and HiSeq X). ${ }^{26} 27$ We aligned reads to the Genome Reference Consortium human genome build 37 (GRCh37) for the earlier participants recruited and GRCh38 for later participants by using Isaac Genome alignment software. Family based variant calling of single nucleotide variants and insertion-deletions for chromosomes 1-22 and $\mathrm{X}$ and the mtDNA used the Platypus variant caller, ${ }^{28}$ allowing joint variant calling for all family members and considering the sequence alignments from all family members together. Figure 1 shows the analysis workflow.

Nuclear variant analysis-single nucleotide variants and small insertion-deletion variants

We analysed genomes in families and classified variants into four "tier" groups according to the probability of the variant being causative. ${ }^{21}$ Tier 1 included loss of function variants (nonsense variants, essential splice donor variants, and essential splice acceptor variants) and de novo missense or splice region variants in genes on the panels applied. Tier 2 included missense and splice region variants in genes on the panels applied. Tier 3 included other rare variants, and a final group of unclassified variants (tier
4) had higher population frequency or the segregation pattern in the family was not consistent with the family history. We chose virtual gene panels according to each participant's phenotypes, using curated "PanelApp" gene lists, which include causative genes for each disorder generated through crowdsourcing. ${ }^{29}$ This allowed the prioritisation of variants likely to be causative and minimised the reporting of abnormal genetic results that are unrelated to the reason for testing-for example, cancer predisposition genes (referred to as incidental findings). All participants had the mitochondrial panel applied and further panels depended on the phenotypes. Tier 1-3 variants were accessed from the Main Programme v8_2019-1128. All tiered variants had passed in-house Genomics England quality control. ${ }^{30}$ We also prioritised variants by using Exomiser, ${ }^{31}$ an application that prioritises variants in exome or genome data by using protein interaction networks, clinical relevance, and cross species phenotype comparisons, as well as computational filters for variant frequency, predicted pathogenicity, and pedigree information. We extracted variants classified as pathogenic, likely pathogenic, or pathogenic/likely pathogenic from Clinvar ${ }^{32}$ (3.3.2020) for GRCh38 and GRCh37 and used bedtools intersect (https://bedtools.readthedocs.io) to compare them against tier 1-3 variants to identify previously reported pathogenic and likely pathogenic variants.

\section{Gene panels applied}

We applied the mitochondrial disorders panel in all participants. Other panels applied included undiagnosed metabolic disorders (148 participants), intellectual disability (139), congenital myopathy (77), and hereditary ataxia (60) (see supplementary table D). The mean number of panels applied per participant was 4.7 (range 1-18). A total of 93 different panels were applied to between one and 345 participants.

\section{Nuclear variant analysis-copy number variants}

We detected copy number variant calls by using Canvas software, ${ }^{33}$ on the basis of sequence coverage and both

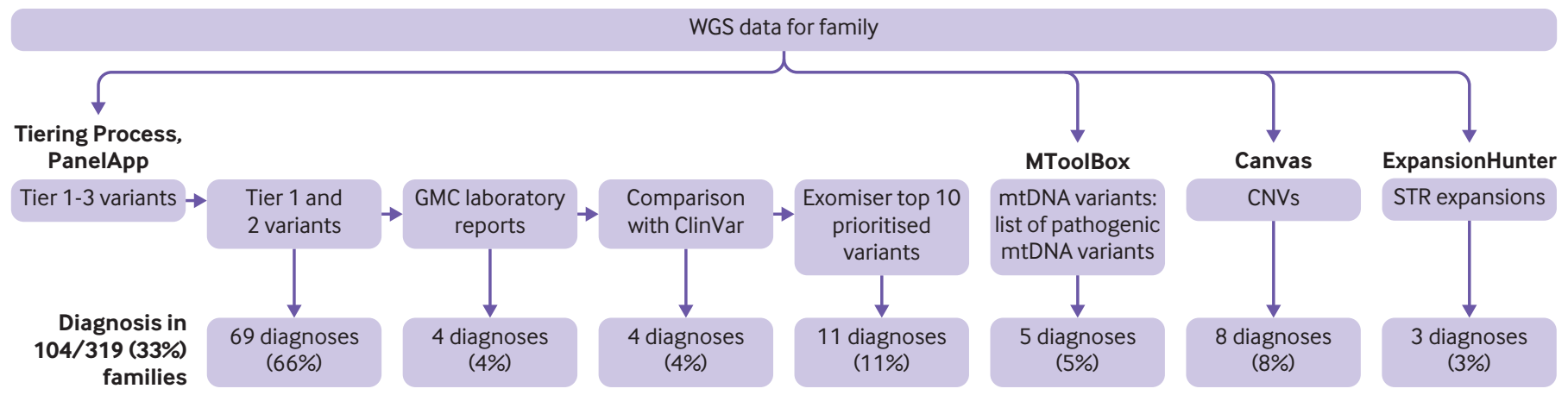

Fig 1 | Overview of analyses and sources of diagnoses. Variants in nuclear genes were analysed using Genomics England tiering system. All tier 1 and tier 2 variants were reviewed, and these provided $66 \%$ of diagnoses. Another $20 \%$ of diagnoses were based on feedback from Genomic Medicine Centre (GMC) laboratories, comparison with Clinvar pathogenic and likely pathogenic variants, and review of top 10 Exomiser prioritised variants. Mitochondrial DNA (mtDNA) variants were analysed separately using in-house pipeline and comparison against list of 89 pathogenic variants, yielding another 5 (5\%) diagnoses. Copy number variants (CNVs) accounted for $8 \%$ of diagnoses and short tandem repeat (STR) expansions for $3 \%$. WGS=whole genome sequencing 
nucleotide and insertion-deletion variant calling. We included only copy number variant calls that had PASS filter status assigned by Canvas and overlapped any gene in PanelApp. We used gencode v29 to annotate copy number variant calls. We evaluated copy number variants that interrupted exons of a PanelApp gene. We manually confirmed all the copy number variants reported as a diagnosis on Integrative Genomics Viewer.

\section{Nuclear variant analysis-short tandem repeat expansions}

We used the ExpansionHunter version 3.2.2 software package for short tandem repeat expansion genotyping. ${ }^{34}$ We assessed 13 loci (HTT, AR, ATN1, ATXN1, ATXN2, ATXN3, ATXN7, CACNA1A, TBP, C9orf72, FXN, FMR1, and DMPK) by using the coordinates listed in supplementary table E. We visually inspected potentially causative repeat expansions above an established threshold for each locus (supplementary table F) by using pileup plots and re-classified them on the basis of the quality of the reads. $^{34}$

\section{mtDNA variant analysis}

We used an in-house pipeline to call mtDNA single nucleotide variants above an established detection threshold of $1 \%$ variant allele frequency (or percentage heteroplasmy level), ${ }^{35}$ after excluding likely errors. ${ }^{27}$ We compared these with a manually curated list of pathogenic mutations with functional evidence supporting pathogenicity. ${ }^{36}$ Our pipeline does not detect large scale mtDNA rearrangements, which usually require targeted mtDNA analysis in DNA extracted from skeletal muscle.

\section{Clinical review-single nucleotide variants and small insertion-deletion variants}

A clinical geneticist reviewed tier 1 and tier 2 variants, the top 10 prioritised variants by Exomiser, ClinVar pathogenic/likely pathogenic variants, and the mtDNA variants (tier 1-3 and from the in-house pipeline) and classified them using internationally accepted criteria for pathogenic variants, likely pathogenic variants, variants of uncertain clinical significance, and likely benign or benign variants (American College of Medical Genetics criteria ${ }^{37}$ ), incorporating information from gnomAD, ${ }^{38}$ ensembl, ${ }^{39}$ VarSome, ${ }^{40}$ OMIM, and a review of the literature (see supplementary methods for a description of the online resources used). Variant quality and variant allele frequency were checked using Integrative Genomics Viewer. ${ }^{41}$

\section{Clinical review-copy number variants}

We reviewed copy number variants that overlapped at least one exon in a PanelApp gene in DECIPHER. We reviewed information about the type of variant (copy number loss, copy number gain, or loss of heterozygosity), the size of the variant, and the gene content (in particular, haploinsufficient genes and OMIM morbid genes). We also made a comparison with previously observed copy number variants in the general population or in affected individuals.

\section{Clinical review-short tandem repeat expansions}

We reviewed participants with repeat expansions to see whether these were causative, considering the size of the expansion (whether it was a "pre-mutation" or "full mutation") and the participant's age and phenotypic features.

\section{Feedback from Genomic Medicine Centre laboratories and clinical teams}

The Genomic Medicine Centre laboratories were commissioned to analyse the tier 1 and tier 2 variants. They filled out an "exit questionnaire" for each family through an online questionnaire. For each family, they fed back whether genetic cause had been identified, with options of "solved," "partial," "uncertain," or "no," and whether the result was already known through clinical testing. For variants that the clinical scientists had identified as potentially causative, they fed back their variant classification according to the American College of Medical Genetics criteria and whether or not the variant had been confirmed by Sanger sequencing and fed back to the clinician. Feedback from the Genomic Medicine Centre laboratories was available in 277/319 (87\%) families (data release v12_21_05_06). We successfully contacted clinicians for further information in 55 (17\%) families.

\section{Overall clinical assessment}

We described the molecular diagnosis as "definite," "probable," or "possible" on the basis of our overall clinical assessment of whether the variant(s) explained the clinical features, taking into account the American College of Medical Genetics classification of the variant(s), the inheritance pattern, and the clinical fit between the patient's HPO terms and the reported clinical phenotypes for the gene or variant. For example, we used "probable" for compound heterozygotes for which the phenotype fitted with the disorder, but we classified only one variant as pathogenic/likely pathogenic and the other was a variant of uncertain clinical significance. We described the contribution as "full" or "partial" depending on whether the whole phenotype or only one aspect could be explained by the variant(s). We classified nuclear genes as "mitochondrial" on the basis of the curated PanelApp list, ${ }^{29}$ plus recently discovered genes known to have a direct effect on oxidative phosphorylation.

\section{Statistical analyses}

Statistical analyses were performed in R. We used Fisher's exact test to compare the number of participants with a genetic diagnosis between children and adults and between singletons and trios/quads. We used Student's $t$ test to compare the mean number of HPO terms in individuals with and without a diagnosis and the number of HPO systems affected. We used Fisher's exact test to compare the proportion of families with each inheritance pattern between nuclear-mitochondrial 
diagnoses and non-mitochondrial disorders. We compared the proportion of participants with each HPO system affected between participants with definite mitochondrial diagnoses and definite non-mitochondrial diagnoses, using Fisher's exact test. We compared the mean Mitochondrial Diagnostic Criteria score between participants with mitochondrial diagnoses, nonmitochondrial diagnoses, and no diagnosis by using a one way analysis of variance and post-hoc Tukey testing.

\section{Patient and public involvement}

The 100000 Genomes Project has a Participant Panel made up of participants and parents or carers of people involved in the project, which was established in 2016. The panel meets with senior staff from Genomics England and NHS England four times a year. They are asked about project design and help to ensure that participants' health data are looked after with respect and used in the best interests of the participants. Panel members sit on other committees including the Access Review Committee and the Ethics Advisory Committee. Patients and carers were involved in developing the consent literature and the infographics and information for the public.

\section{Results}

\section{Demographics}

Three hundred and forty five affected individuals (186 females, 159 males) were referred with a suspected mitochondrial disorder after exclusion of common causes. They were from 319 families of different reported ethnicities across England (fig 2). Genomic data were available for more than one affected member in 25 families (15 sibling pairs, eight mother and child pairs, one father and child pair, and one mother with two affected children). The median age at enrolment for probands was 25 (interquartile range 10-54; range 0-92) years; 143 (41\%) were aged $\leq 18$ at enrolment. No participants withdrew or were lost to follow-up.

\section{Phenotype data}

Phenotypic data were available for 341 participants (missing in four participants). A total of 3095 HPO terms were recorded, with a median of 7 (range 1-39) HPO terms per participant; 806 different HPO terms were used. Figure 3 shows the most common clinical terms, investigation result terms, and HPO systems affected. A median of 4 (range 1-13) HPO systems were affected per participant. Application of the HPO modified Nijmegen score gave a mean total score of 4.30 (range 0-10), with 24/345 (7\%) participants classified as being unlikely to have mitochondrial disease (score 0 or 1), $193 / 345(56 \%)$ as Nijmegen possible (score 2-4), 95/345 (28\%) as Nijmegen probable (score 5-7), and $33 / 345(10 \%)$ as Nijmegen definite (score of $\geq 8$ ).

\section{Diagnostic yield}

We identified the definite or probable genetic diagnosis in 98/319 (31\%) families, with a possible diagnosis in an additional six (2\%) (table 1; table 2; fig 1; fig 4). A definite genetic diagnosis was reached in 28\% (89/319), including 14 (4\%) genetic diagnosis that provided only a partial explanation for their clinical features. Nine (3\%) families had a probable diagnosis, and six (2\%) had a possible diagnosis. One participant was diagnosed as having two disorders that together fully explained the combination of phenotypes. Most diagnoses (69/104; $66 \%$ ) came from single nucleotide variants or small insertion-deletion variants on the panels applied (tier 1 or tier 2 variants). An additional 19/104 (18\%) diagnoses were made through other analyses of the single nucleotide variants and small insertion-deletion variants (from the Genomic Medicine Centre laboratory reports, comparison with ClinVar, and Exomiser). The copy number variant analysis added eight (8\%) diagnoses, and the short tandem repeat analysis added three (3\%) diagnoses. Five (5\%) diagnoses were made through the mtDNA analysis. Further details of the variant analysis are shown in supplementary table G.

\section{Factors affecting diagnostic yield}

The overall diagnostic rate was 59/186 (32\%) in females and 51/159 (32\%) in males. Sixty four (45\%) of 143 participants recruited aged 18 or under received a diagnosis (any type), compared with 46/202 (23\%) participants over the age of $18(\mathrm{P}<0.001)$. Considering definite diagnoses only, 50/143 (35\%) participants under 18 received a definite molecular diagnosis compared with 30/202 (15\%) adults over the age of 18 $(\mathrm{P}<0.001)$. Figure 5 shows the age profile for diagnosis. Although diagnoses were more frequent in younger people, diagnoses were still being made in participants who were in their 70s or 80s at enrolment.

The diagnostic yield per family (all diagnoses) was 23\% (23/102) for singletons, 29\% (12/42) for duos with a parent, $42 \%(62 / 148)$ for trios/quads, and $26 \%$ (7/27) for other family structures. The diagnostic rate in trios/quads was higher than in singletons $(\mathrm{P}=0.005)$. The mean age of singletons was 56 years, compared with a mean age of 12 years for probands in trios/quads.

The mean number of HPOs in participants with any diagnosis (10.0) was slightly higher than in patients with no diagnosis (8.48) $(\mathrm{P}=0.03)$. The mean number of HPO systems affected in patients with a diagnosis (4.67) was not significantly different from the mean number of HPO systems affected in those with no diagnosis (4.61) $(\mathrm{P}=0.82)$.

\section{Types of diagnosis}

Of the 75 families with a definite diagnosis that fully explains the phenotype, 28 (37\%) were in genes known to cause primary mitochondrial disease, including four mtDNA variants and 24 diagnoses in nuclear-mitochondrial genes, whereas 47 (63\%) were in non-mitochondrial genes. For families with any genetic diagnosis (including probable or possible diagnoses and partial explanations), 39/104 (38\%) were in genes known to cause primary mitochondrial disease, including six mtDNA variants and 30 nuclearmitochondrial diagnoses, and $65(63 \%)$ were in non-mitochondrial genes. These non-mitochondrial diagnoses included a wide variety of disorders, including developmental disorders with intellectual disability, 

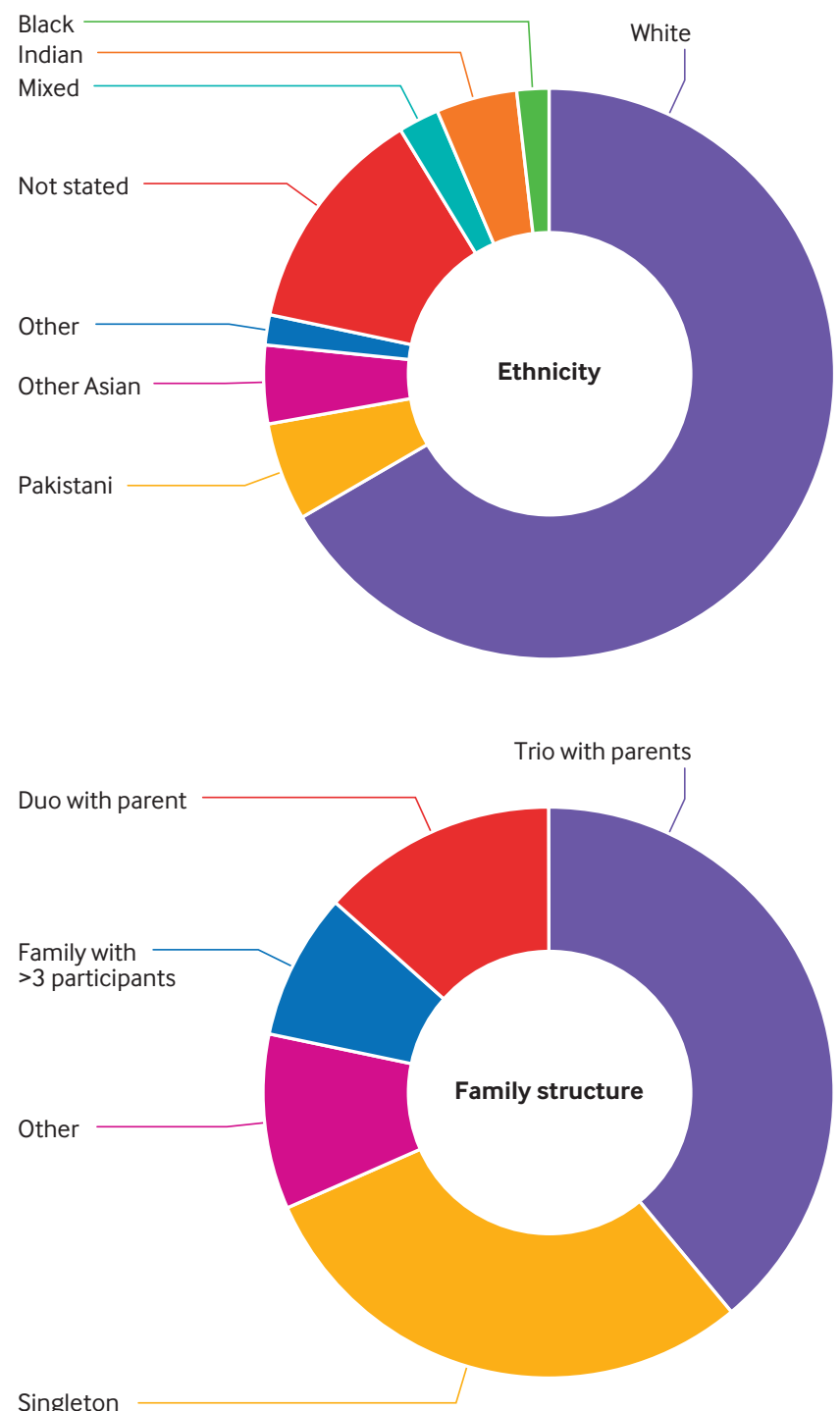

Singleton

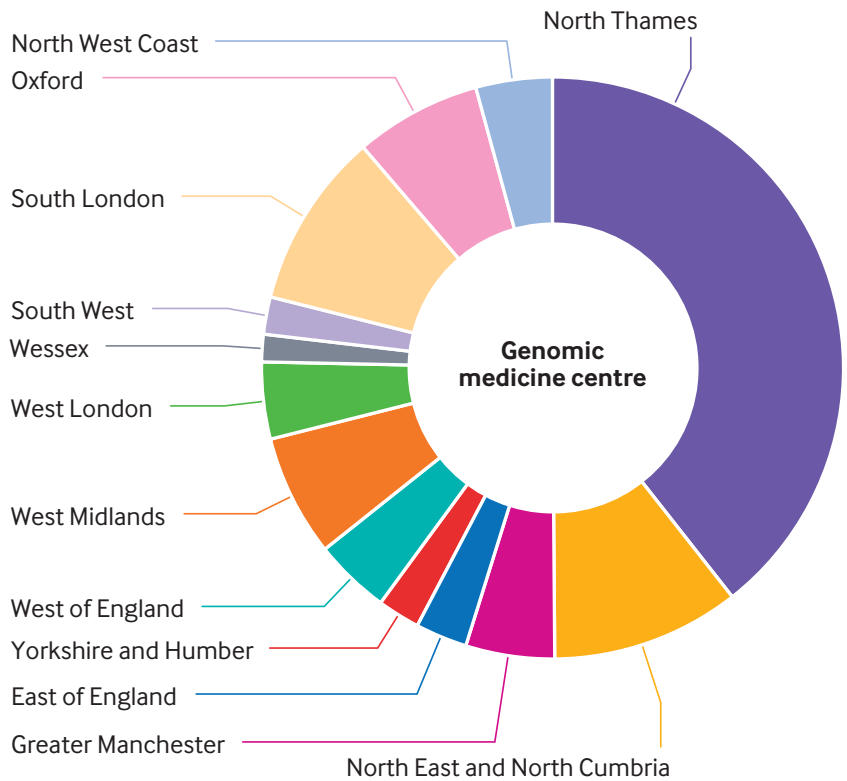

Fig 2 | Demographics for participants recruited. Top: ethnicities recorded in participants reflected ethnicity of overall population in England. Middle: most commonly recruited family structures were trios with both parents and singletons. Bottom: participants were recruited from Genomic Medicine Centres across England 

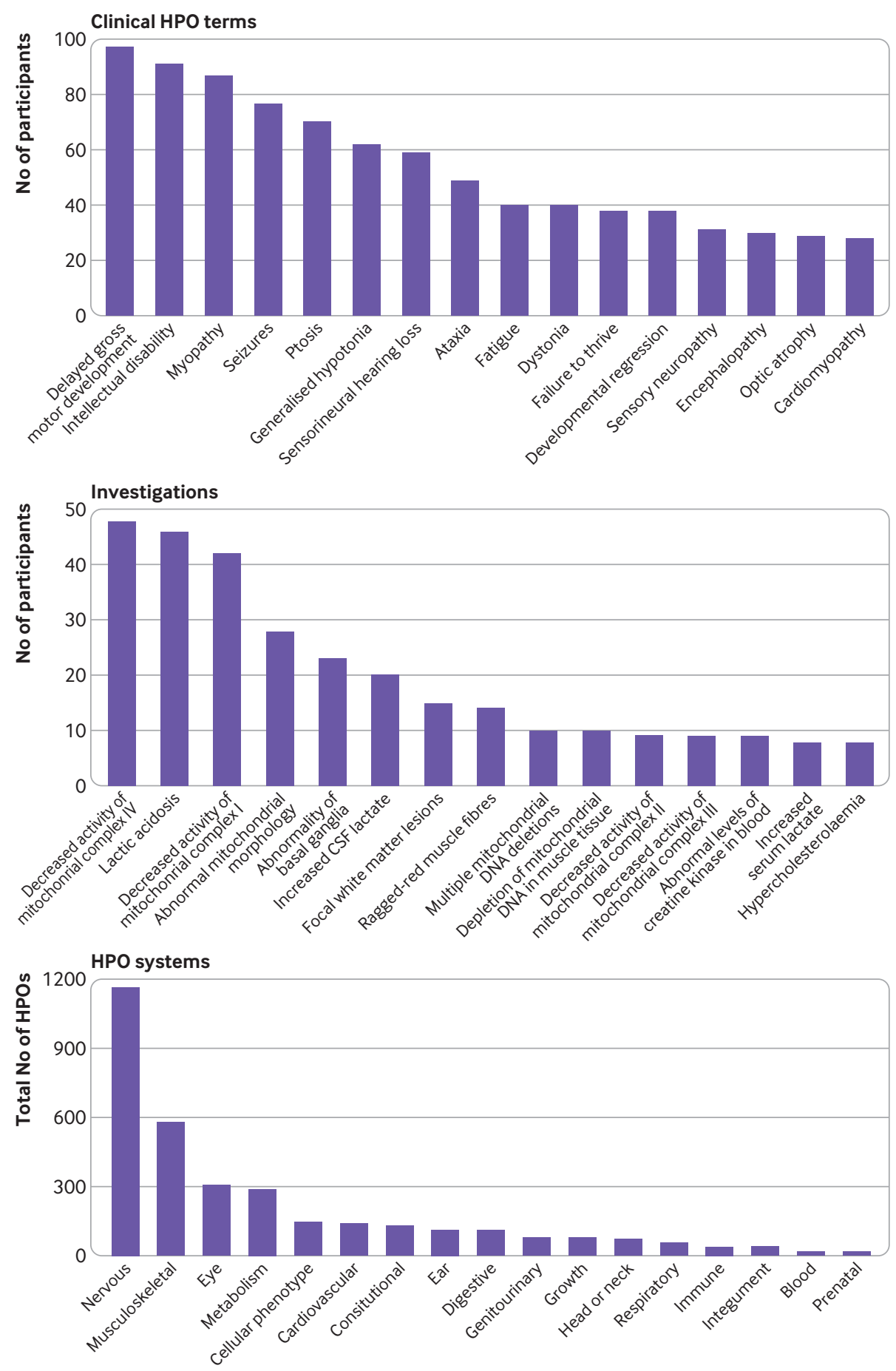

Fig 3 | Human Phenotype Ontology (HPO) terms for participants recruited. Top: most commonly recorded clinical HPO terms, including delayed gross motor development, intellectual disability, and myopathy. Middle: most commonly recorded investigation results HPO terms, including decreased activity of mitochondrial complex IV, lactic acidosis, and decreased activity of mitochondrial complex I. Bottom: total number of HPO terms recorded for 345 participants according to ancestor HPO system (some HPO terms have more than one ancestor HPO system)

metabolic disorders, epileptic encephalopathies, Bardet-Biedl syndrome, cardiomyopathies, MYH2related myopathy, and amyloidosis.

Potentially treatable disorders

Potentially treatable disorders were identified in six participants with a mitochondrial disorder and nine participants with a non-mitochondrial disorder (shown in supplementary table $\mathrm{H}$ ). However, the evidence base is weak for several of these rare disorders. ${ }^{42}$

Mitochondrial diagnoses compared with nonmitochondrial diagnoses

Inheritance patterns

Seventy per cent $(23 / 33)$ of families with a nuclearmitochondrial diagnosis showed an autosomal 


\begin{tabular}{|c|c|c|c|c|c|c|c|}
\hline Family & Age, years & Sex & Contribution & Gene & Variants & ACMG & Inheritance \\
\hline \multicolumn{8}{|c|}{ Definite } \\
\hline 1 & 10 & $\mathrm{~F}$ & Full & AARS2 & NM_020745.4:c.302G >A p.(Arg101His); homozygous & LP & Biallelic \\
\hline 2 & 1 & M & Full & AIFM1 & NM_004208.4: c.603_605del p.(Arg201del) & $\mathrm{P}$ & $\mathrm{XLR}$; de novo \\
\hline 3 & 0 & $\mathrm{~F}$ & Full & ATAD3 & Duplication in ATAD3 gene cluster & $\mathrm{P}$ & Monoallelic; de novo \\
\hline 4 & 0 & M & Full & ATAD3 & Duplication in ATAD3 gene cluster & $\mathrm{P}$ & Monoallelic; de novo \\
\hline \multirow[t]{2}{*}{5} & \multirow[t]{2}{*}{13} & \multirow[t]{2}{*}{ M } & \multirow[t]{2}{*}{ Full } & \multirow[t]{2}{*}{ C12orf65 } & NM_152269.5:c.210del p.(Gly72fs) & $\mathrm{P}$ & \multirow[t]{2}{*}{ Biallelic } \\
\hline & & & & & NM_152269.5:c.258_270dupCATCCCCTCAGGC p.(Ile91fs)* & $\mathrm{P}$ & \\
\hline 6 & 6 & M & Full & EARS2 & NM_001083614.2:c.184A >T p.(Ile62Phe); homozygous & $\mathrm{LP}$ & Biallelic \\
\hline \multirow[t]{2}{*}{7} & \multirow[t]{2}{*}{2} & \multirow[t]{2}{*}{$\mathrm{F}$} & \multirow[t]{2}{*}{ Full } & \multirow[t]{2}{*}{$F B X L 4$} & NM_001278716.2:c.1641_1642delTG p.(Cys547Ter) & $\mathrm{P}$ & \multirow[t]{2}{*}{ Biallelic } \\
\hline & & & & & NM_001278716.2:c.141delC p.(Asn48fs) * & $\mathrm{P}$ & \\
\hline 8 & 18 & $\mathrm{~F}$ & Full & $\mathrm{HIBCH}$ & NM_014362.4:C.1126T >G p.(Phe376Val)*; homozygous & $\mathrm{LP}$ & Biallelic \\
\hline \multirow[t]{2}{*}{9} & \multirow[t]{2}{*}{22} & $\mathrm{~F}$ & Full & KARS1 & NM_001130089.1:c.683C >T p.(Pro228Leu) & $\mathrm{P}$ & Biallelic \\
\hline & & & & & NM_001130089.1:c.774A >T p.(Arg258Ser) & LP & \\
\hline 10 & 1 & $\mathrm{~F}$ & Full & MRPL44 & NM_022915.4:c.467T >G p.(Leu156Arg); homozygous & $\mathrm{P}$ & Biallelic \\
\hline 11 & 23 & M & Full & MRPS25t & NM_022497.5:c.215C >T p.(Pro72Leu); homozygous & LP & Biallelic \\
\hline 12 & 42 & $\mathrm{~F}$ & Full & MT-ATP6 & m.8618dupT; $14 \%$ heteroplasmy & $\mathrm{P}$ & mtDNA; very low level in mother \\
\hline 13 & 24 & $M$ & Full & MT-ATP6 & m.8969G >A; $84 \%$ heteroplasmy & $P$ & mtDNA; de novo \\
\hline 14 & 26 & $\mathrm{~F}$ & Full & MT-ND3 & m.10158T >C; 23\% heteroplasmy & $P$ & mtDNA; de novo \\
\hline 15 & 67 & $M$ & Partial & MT-ND6 & m.14484T >C; homoplasmic & $P$ & mtDNA; unknown \\
\hline 16 & 18 & $M$ & Full & MT-TE & m.14674T >C; homoplasmic & $P$ & mtDNA; maternally inherited \\
\hline 17 & 18 & $\mathrm{~F}$ & Partial & MT-RNR1 & m.1555A >G; homoplasmic & $P$ & mtDNA; maternally inherited \\
\hline 18 & 10 & $\mathrm{~F}$ & Full & MTO1 & NM_012123.4:c.1232C >T p.(Thr411lle); homozygous & $P$ & Biallelic \\
\hline 19 & 13 & $\mathrm{~F}$ & Full & NDUFAF5 & NM_024120.5:c.480-3T >G* & $\mathrm{LP}$ & Biallelic \\
\hline & & & & & NM_024120.5:c.827G >A p.(Arg276GIn)* & $L P$ & \\
\hline 20 & 1 & M & Full & NDUFAFB & NM_001086521.2:c.45_52dup p.(Phe18fs) & $P$ & Biallelic \\
\hline & & & & & NM_001086521.2:C.195+271C >T & LP & \\
\hline 21 & 7 & M & Full & OPA1 & NM_015560.2:c.2708_2711delTTAG (splice acceptor variant) & $P$ & Biallelic \\
\hline & & & & & NM_015560.2:c.1146A >G p.(Ile382Met) & $P$ & \\
\hline 22 & 11 & $\mathrm{~F}$ & Full & PDHA1 & NM_000284.4:c.434G >A p.(Cys145Tyr)* & LP & XLD; de novo \\
\hline 23 & 17 & $\mathrm{~F}$ & Full & PDP1 & NM_018444.4:c.571C >T p.(GIn191Ter) ${ }^{*}$ homozygous & LP & Biallelic \\
\hline & 13 & $\mathrm{~F}$ & & & & & \\
\hline 24 & 56 & M & Full & POLG & NM_002693.3:c.1399G >A p.(Ala467Thr); homozygous & $P$ & Biallelic \\
\hline 25 & 48 & $\mathrm{~F}$ & Full & RRM2B & NM_001172477.1:c.242G >A p.(Asp142Asn) & $P$ & Monoallelic; AD \\
\hline & 71 & $\mathrm{~F}$ & & & & & \\
\hline 26 & 0 & M & Full & $\mathrm{SCO} 2$ & NM_001169109.1:c.418G >A p.(Glu140Lys)* & $\mathrm{P}$ & Biallelic \\
\hline & & & & & NM_001169109.1:c.625_627delTAC p.(Tyr209del)* & $L P$ & \\
\hline 27 & 0 & $\mathrm{~F}$ & Full & $\mathrm{SCO} 2$ & NM_001169109.1:c.323A >G p.(Asp108Gly) & $L P$ & Biallelic \\
\hline & & & & & NM_001169109.1:c.281T >C p.(Leu94Pro) ${ }^{\star}$ & $L P$ & \\
\hline 28 & 74 & $\mathrm{~F}$ & Full & SLC25A4 & NM_002252.4:c.311A >G p.(Asp104Gly) & $\mathrm{P}$ & Monoallelic; unknown \\
\hline 29 & 20 & $\mathrm{~F}$ & Full & TTC19 & NM_017775.4:C.184+1G >A* & $P$ & Biallelic \\
\hline & & & & & NM_017775.4:C.275_278delCCGA p.(Ala92fs)* & $\mathrm{LP}$ & \\
\hline 30 & 66 & M & Full & TWNK & NM_021830.5:c.1374G >T p.(Gln458His) & $\mathrm{P}$ & Monoallelic; unknown \\
\hline Probab & & & & & & & \\
\hline 31 & 61 & M & Full & DNM1L & NM_012062.5:c.239_241delGAG p.(Gly80del)* & LP & Monoallelic; unknown \\
\hline 32 & 4 & $\mathrm{~F}$ & Full & ELAC2 & NM_018127.7:c.2009delG p.(Cys670fs) & $\mathrm{P}$ & Biallelic \\
\hline & & & & & NM_018127.7:c.2245C >T p.(His749Tyr)* & VUS & \\
\hline 33 & 7 & $\mathrm{~F}$ & Full & GFER & NM_005262.3:c.199delC p.(Arg67fs) & $\mathrm{P}$ & Biallelic \\
\hline & & & & & NM_005262.3:C.259-28C >G* & VUS & \\
\hline 34 & 19 & M & Full & MTFMT & NM_139242.4:c.626C >T p.(Ser209Leu) & $\mathrm{P}$ & Biallelic \\
\hline & & & & & NM_139242.4:C721+5G $>A^{*}$ & VUS & \\
\hline 35 & 4 & $\mathrm{~F}$ & Full & RRM2B & NM_001172477.1:c.578G >A p.(Arg193His) & LP & Biallelic \\
\hline & & & & & NM_001172477.1:c1253C >A p.(Thr418Asn)* & VUS & \\
\hline 36 & 3 & M & Full & SDHA & NM_004168.4:c.290G >C p.(Arg97Thr)* & VUS & Biallelic \\
\hline & & & & & NM_004168.4:c.424A >G p.(Met142Val)* & VUS & \\
\hline Possib & & & & & & & \\
\hline 37 & 18 & M & Full & LONP1 & NM_004793.4: c.1694A >G p.(Tyr565Cys) & VUS & De novo \\
\hline 38 & 0 & M & Full & PDHA1 & NM_000284.4: C.759+5G >T & VUS & $\mathrm{XL}$ (from unaffected mother) \\
\hline 39 & 56 & M & Full & TOP3A & NM_004618.5:c.284C >T p.(Ala95Val)* & VUS & Presumed biallelic (parents not tested) \\
\hline & 54 & $\mathrm{~F}$ & & & NM_004618.5:c.109C >G p.(Leu37Val)* & VUS & \\
\hline $\begin{array}{l}\text { Genetic } \\
(\mathrm{ACMG}) \mathrm{v} \\
\mathrm{AD}=\text { auto } \\
{ }^{*} \text { Novel va } \\
\text { +Previous }\end{array}$ & $\begin{array}{l}\text { agnoses made } \\
\text { riant classificat } \\
\text { omal dominant } \\
\text { iants } \\
\text { y published fan }\end{array}$ & $\begin{aligned} & 39 \mathrm{fam} \\
& \text { on }(P=p \\
&==\text { femal }\end{aligned}$ & $\begin{array}{l}\text { milies, includin } \\
\text { oathogenic, } L P= \\
\text { le; } M=\text { male; } m\end{array}$ & $\begin{array}{l}\text { ge and sex of } \\
\text { ely pathogen } \\
V A=\text { mitochon }\end{array}$ & $\begin{array}{l}\text { articipants, contribution to phenotype from this gene (full or partial), ger } \\
\text { VUS =variant of uncertain clinical significance), and inheritance pattern. } \\
\text { rial DNA; } X L=X \text { linked; } X L D=X \text { linked dominant; } X L R=X \text { linked recessive. }\end{array}$ & variants & American College of Medical Genetics \\
\hline
\end{tabular}

recessive inheritance pattern, compared with 35\% $(23 / 65)$ of families with non-mitochondrial disorders. Five $(15 \%)$ families had de novo diagnostic variants in nuclear-mitochondrial genes (AIFM1, LONP1, and PDHA1 and two with de novo duplications in the ATAD3 gene cluster) compared with 29\% (19/65) of 
families with non-mitochondrial disorders having a de novo diagnosis (fig 6), particularly those with intellectual disability or epileptic encephalopathy. The HK1 variant was de novo in two siblings with presumed germline mosaicism (somatic mosaicism was not detected in either parent). The proportions with each inheritance pattern were significantly different between nuclear-mitochondrial diagnoses and non-mitochondrial disorders $(\mathrm{P}=0.007)$.

\section{HPO systems affected}

In our cohort, participants with a mitochondrial diagnosis were more likely to have HPO terms in the metabolism/homoeostasis system $(\mathrm{P}<0.001)$, such as increased lactate or decreased mitochondrial complex activities (fig 7), than were those with nonmitochondrial diagnoses (fig 8). Otherwisw, the pattern of HPO systems involved was similar between the two groups.

Modified Nijmegen Mitochondrial Disease Criteria scores Most (22/30; 73\%) participants with genetically confirmed mitochondrial diagnoses (nuclearmitochondrial and mtDNA) had scores in the Nijmegen probable (5-7) and Nijmegen definite (8-12) modified Nijmegen Mitochondrial Disease Criteria category (fig 9), whereas $60 \%(30 / 50)$ of participants with confirmed non-mitochondrial disorders had scores in the possible range (score 2-4). The mean Nijmegen Mitochondrial Disease Criteria score differed between diagnostic groups $(\mathrm{P}<0.001)$, including between the mitochondrial diagnoses and the non-mitochondrial diagnoses $(\mathrm{P}<0.001)$ and between the mitochondrial diagnoses and patients with no diagnosis $(\mathrm{P}<0.001)$ but not between non-mitochondrial diagnoses and patients with no diagnosis $(\mathrm{P}=0.36)$. However, $16 / 50$ (32\%) participants with confirmed non-mitochondrial disorders had scores in the Nijmegen probable range, and three $(6 \%)$ had scores in the Nijmegen definite range. The sensitivity and specificity of the Nijmegen Mitochondrial Disease Criteria score in our cohort are shown in supplementary table I. Using Nijmegen definite, the score was highly specific but sensitivity was only $40 \%$, whereas using Nijmegen probable and definite scores, the score was moderately sensitive and moderately specific.

\section{Muscle biopsy findings and mitochondrial complex activities}

One hundred and seventeen (34\%) participants had HPO terms relating to muscle biopsy abnormalities, and $73(21 \%)$ had abnormal mitochondrial complex activities. Of patients with definite mitochondrial diagnoses, $15 / 28$ (54\%) had muscle biopsy abnormalities, 11/28 (39\%) had abnormal respiratory chain complex activities, and two had abnormal pyruvate dehydrogenase complex activity. Of patients with definite non-mitochondrial diagnoses, 14/47 (30\%) had muscle biopsy abnormalities and 12/47 (26\%) had abnormal respiratory chain complex activities. The patients with non-mitochondrial diagnoses and abnormal respiratory chain complex activities had pathogenic variants in ASXL3, CACNA1E, CTBP1, EXOSC3, HK1, KCNT1, NPHP1, P4HTM, PPP2R5D and SCN2A, SOS1, and TANGO2.

\section{Discussion}

In patients referred for whole genome sequencing with a suspected mitochondrial disorder, a definite or probable diagnosis was identified in 31\% (including partial diagnosis in 4\%) and a further $2 \%$ had a possible diagnosis. The mitochondrial diagnoses were nearly all unique to one family, reflecting the high level of genetic heterogeneity in mitochondrial disorders. Non-mitochondrial disorders were more common than mitochondrial disorders and had features resembling mitochondrial diseases (often referred to as phenocopies). These could be broadly classified as developmental disorders with intellectual disability, metabolic disorders, myopathies, cardiomyopathies, epileptic encephalopathies, leukodystrophies, ciliopathies, amyloidosis, and other neurogenetic disorders, including basal ganglia calcification and neurodegeneration with iron accumulation. Of the non-mitochondrial disorders, $29 \%$ were caused by de novo pathogenic variants.

The diagnostic yield was significantly greater in children than in adults. Several possible explanations for this exist. Firstly, children were more likely to be recruited as trios with both parents. This makes analysis more straightforward because de novo variants can be identified, rare familial variants can be filtered out if the parents are clinically unaffected for recessive disorders, and it is possible to check that the variants were inherited from both parents..$^{43}$ Secondly, the most severe phenotypes are seen in children because affected individuals do not survive until adulthood..$^{44}$ Severe phenotypes are the most likely to be caused by single gene disorders. Milder phenotypes overlap with acquired disorders, are less likely to be caused by a single gene defect, and are more common in adults. Thirdly, most adults with genetically proven mitochondrial disorders have mutations in the mtDNA that were excluded by the clinical laboratories before inclusion in this study. Studies of whole exome sequencing in rare disease have also noted a decrease in diagnostic yield with increasing age of the probands. ${ }^{45}$ Despite this, in our study, new genetic diagnoses were made across the whole age spectrum. The oldest patient to receive a genetic diagnosis was recruited at age 86 .

The number of people recruited in different age groups was also variable. The highest numbers were recruited in the paediatric age group, with a second peak of patients aged over 60 years. This could be because some adult onset mitochondrial disorders, such as progressive external ophthalmoplegia, take time to develop and become obvious only in later life (progressive external ophthalmoplegia was present in $17 / 71$ (24\%) over 60s compared with $16 / 274(6 \%)$ under 60s). Alternatively, this could reflect the fact that genetic testing had not been offered previously to the 
Table 2 | Variants identified in patients with definite, probable, and possible non-mitochondrial diagnoses

\begin{tabular}{|c|c|c|c|c|c|c|c|}
\hline Family & $\begin{array}{l}\text { Age, } \\
\text { years }\end{array}$ & Sex & Contribution & Gene & Variants & ACMG & Inheritance \\
\hline \multicolumn{8}{|c|}{ Definite } \\
\hline 40 & 0 & $\mathrm{~F}$ & Full & ACTA2 & NM_001614.4:C.536G >A p.(Arg179His) & $\mathrm{P}$ & Monoallelic; de novo \\
\hline \multirow[t]{2}{*}{41} & \multirow[t]{2}{*}{42} & \multirow[t]{2}{*}{$\mathrm{F}$} & \multirow[t]{2}{*}{ Full } & \multirow[t]{2}{*}{$A M A C R$} & NM_014324.6:c.857delT p.(Ile286fs)* & $\mathrm{P}$ & \multirow[t]{2}{*}{ Biallelic } \\
\hline & & & & & NM_014324.6:c.437C >T p.(Pro146Leu) ${ }^{*}$ & $\mathrm{LP}$ & \\
\hline 42 & 57 & M & Full & AMACR & NM_014324.6:c.154T >C p.(Ser52Pro); homozygous & $\mathrm{P}$ & Biallelic \\
\hline 43 & 71 & $\mathrm{~F}$ & Full & AMACR & NM_014324.6:c.154T >C p.(Ser52Pro); homozygous & $\mathrm{P}$ & Biallelic \\
\hline 44 & 9 & $\mathrm{~F}$ & Full & AMPD2 & NM_001368809.2: c.2228T >C p.(Leu743Pro)*; homozygous & $L P$ & Biallelic \\
\hline \multirow[t]{2}{*}{45} & 29 & M & \multirow[t]{2}{*}{ Full } & \multirow[t]{2}{*}{ APP } & \multirow[t]{2}{*}{ NM_000484.4:c.2075C >G p.(Ala692Gly) } & \multirow[t]{2}{*}{$\mathrm{P}$} & Monoallelic; AD \\
\hline & 58 & $\mathrm{~F}$ & & & & & \\
\hline 46 & 18 & $M$ & Partial & $A S L$ & NM_000048.4:c.1153C >T p.(Arg385Cys); homozygous & $P$ & Biallelic \\
\hline 47 & 15 & M & Full & ASXL3 & NM_030632.3:c.3464C >A p.(Ser1155Ter) & $P$ & Monoallelic; de novo \\
\hline 48 & 15 & $M$ & Full & ATP1A3 & NM_152296.5:c.2452G >A p.(Glu818Lys) & $P$ & Monoallelic; de novo \\
\hline 49 & 86 & $\mathrm{~F}$ & Full & ATP1A3 & NM_152296.5:c.2452G >A p.(Glu818Lys) & $\mathrm{P}$ & Monoallelic; unknown \\
\hline 50 & 5 & $M$ & Full & ATP6V1A & NM_001690.4:c.845A >T p.(Asn282lle)* & $L P$ & Monoallelic; de novo \\
\hline 51 & 1 & $\mathrm{~F}$ & Full & ATXN7† & Very large CAG repeat expansion & $P$ & Monoallelic \\
\hline 52 & 44 & $\mathrm{~F}$ & Full & BBS1 & NM_024649.5:c.1169T >G p.(Met390Arg); homozygous & $P$ & Biallelic \\
\hline 53 & 2 & M & Full & BCAP31 & NM_001256447.2:C.565C >T p.(Gly189Ter)* & $P$ & XLR; de novo \\
\hline 54 & 26 & $\mathrm{~F}$ & Full & C19orf12 & NM_001256047.1:c.245dupC p.(Ala83fs) ${ }^{*}$ & $L P$ & Monoallelic; unknown \\
\hline 55 & 12 & $\mathrm{~F}$ & Full & CACNA1A & NM_001127221.1:c.4177G >A p.(Val1393Met) & $L P$ & Monoallelic; de novo \\
\hline 56 & 2 & M & Full & CACNA1E & NM_001205293.3:c.683T >C p.(Leu228Pro) & $\mathrm{LP}$ & Monoallelic; de novo \\
\hline 57 & 12 & $M$ & Full & CTBP1 & NM_001328.3:c.1024C >T p.(Arg342Trp) & $P$ & Monoallelic; de novo \\
\hline 58 & 11 & M & Full & DOCK6 & NM_020812.4:C.4106+5G >T & $\mathrm{LP}$ & Biallelic \\
\hline & 10 & $\mathrm{~F}$ & & & NM_020812.4:c.1902_1905delGTTC p.(Phe635fs) & $\mathrm{P}$ & \\
\hline 59 & 12 & $\mathrm{~F}$ & Full & DSP & NM_004415.4:c.1799T >C p.(Phe600Ser) ${ }^{*}$ & $L P$ & Monoallelic; de novo \\
\hline 60 & 8 & $\mathrm{~F}$ & Full & EXOSC3 & NM_016042.4:c.395A>C p.(Asp132Ala); homozygous & $P$ & Biallelic \\
\hline 61 & 61 & $M$ & Partial & EYA4 & NM_004100.5:c.1741A >T p.(Lys581Ter)* & $L P$ & Monoallelic; unknown \\
\hline 62 & 2 & M & Full & FIG4 & NM_014845.6:c.447-2A >G* & $\mathrm{P}$ & Biallelic \\
\hline & & & & & NM_014845.6:c.827C >T p.(Ser276Phe) ${ }^{\star}$ & $L P$ & \\
\hline 63 & 6 & $\mathrm{~F}$ & Full & GCDH & NM_000159.4:c.1204C >T p.(Arg402Trp) & $P$ & Biallelic \\
\hline & & & & & NM_000159.4:C.1304C >T p.(Thr435Met)* & $\mathrm{LP}$ & \\
\hline 64 & 3 & $\mathrm{~F}$ & Full & HADHA & NM_000182.5:c.1528G >C p.(Glu510GIn) & $P$ & Biallelic \\
\hline & & & & & NM_000182.5:c.1664T >G p.(Met555Arg)* & $L P$ & \\
\hline 65 & 10 & $\mathrm{~F}$ & Full & HK1 & NM_000188.3:c.1334C >T p.(Ser445Leu) & $P$ & Monoallelic; de novo \\
\hline & 8 & $\mathrm{~F}$ & & & & & \\
\hline 66 & 9 & M & Full & HSD17B4 & NM_000414.4:c.590_597dupGATCACGG p.(Met200fs)* & $\mathrm{P}$ & Biallelic \\
\hline & & & & & NM_000414.4:c.743G >A p.(Arg248His)* & LP & \\
\hline 67 & 71 & M & Partial & HTTt & $\sim 40$ CAG repeats & $\mathrm{P}$ & Monoallelic \\
\hline 68 & 7 & $\mathrm{~F}$ & Full & HTTt & Very large CAG repeat expansion & $P$ & Monoallelic \\
\hline 69 & 41 & M & Partial & KCNQ4 & NM_004700.4:c.961G >A p.(Gly321Ser) & $\mathrm{P}$ & Monoallelic; unknown \\
\hline 70 & 6 & $\mathrm{~F}$ & Full & KCNT1 & NM_020822.3:c.1885A >c p.(Lys629GIn) & $L P$ & Monoallelic de novo \\
\hline 71 & 46 & $\mathrm{~F}$ & Full & KIF11 & NM_004523.4:C.78-2A >G* & $\mathrm{P}$ & Monoallelic; AD \\
\hline 72 & 29 & M & Partial & KMT2C & NM_170606.3:c.11669delA p.(Gln3890fs)* & $P$ & Monoallelic; de novo \\
\hline 73 & 0 & $\mathrm{~F}$ & Full & MBD5 & NM_001378120.1 deletion of exon 2 & $L P$ & Monoallelic; unknown \\
\hline 74 & 72 & $M$ & Full & MYH2 & NM_017534.6:c.2116G >A p.(Glu706Lys) & $\mathrm{P}$ & Monoallelic; unknown \\
\hline 75 & 57 & $M$ & Partial & MYH7 & NM_000257.4:c.1357C >T p.(Arg453Cys) & $P$ & Monoallelic; unknown \\
\hline 76 & 13 & $M$ & Full & NARS1 & NM_004539.4:c.1600C >T p.(Arg534Ter) & $\mathrm{P}$ & Monoallelic; de novo \\
\hline 77 & 19 & $\mathrm{~F}$ & Full & NPHP1 & Gene deletion;homozygous & $P$ & Biallelic \\
\hline & 17 & $\mathrm{~F}$ & & & & & \\
\hline 78 & 3 & M & Full & NPHP1 & Gene deletion; homozygous & $P$ & Biallelic \\
\hline 79 & 31 & $\mathrm{~F}$ & Partial & OPTN & Gene deletion & $P$ & Monoallelic; unknown \\
\hline 80 & 1 & M & Full & P4HTM & NM_177939.3:c.659G >A p.(Trp220Ter) ${ }^{*}$ & $\mathrm{P}$ & Biallelic \\
\hline & & & & & NM_177939.3:c.569_579del p.(Gln190fs)* & $\mathrm{P}$ & \\
\hline 81 & 36 & $\mathrm{~F}$ & Full & PDGFB & Gene deletion & $P$ & Monoallelic; AD \\
\hline 82 & 11 & $\mathrm{~F}$ & Partial & PHKB & NM_000293.3:c.2109delT p.(Ser704fs)* & $\mathrm{P}$ & Biallelic \\
\hline & & & & & NM_000293.3:c. $2427+977 C>T^{\star}$ & $\mathrm{P}$ & \\
\hline 83 & 3 & M & Partial & PKD2 & NM_000297.4:c.1390C >T p.(Arg464Ter) & $P$ & Monoallelic; AD \\
\hline 84 & 45 & $\mathrm{~F}$ & Full & $P M M 2$ & NM_000303.3:c.442G >A p.(Asp148Asn) & $\mathrm{P}$ & Biallelic \\
\hline & & & & & NM_000303.3:c.305A >G p.(Tyr102Cys)* & $L P$ & \\
\hline & & & & $A Q P 2$ & NM_000486.6:c.707_720dupTGCTGAAGGGCCTG p.(Glu241fs)* & $L P$ & Biallelic \\
\hline & & & & & NM_000486.6:c.34G >A p.(Ala12Thr)* & $L P$ & \\
\hline 85 & 2 & $\mathrm{~F}$ & Full & POGZ & NM_05100.4:c.2571-2delA* & $P$ & Monoallelic; de novo \\
\hline 86 & 54 & $\mathrm{~F}$ & Full & POLR3A & NM_007055.4:c.2119C >T p.(GIn707Ter) & $P$ & Biallelic \\
\hline & & & & & NM_007055.4:C.1909+22G >A & $\mathrm{P}$ & \\
\hline 87 & 2 & M & Full & PPP2R5D & NM_006245.4:C.592G >A p.(Glu198Lys) & $\mathrm{P}$ & Monoallelic; de novo \\
\hline 88 & 7 & $\mathrm{~F}$ & Full & SAMD9 & NM_001193307.1: c.2053C >T p.(Arg685Ter) & $\mathrm{LP}$ & Monoallelic; de novo \\
\hline 89 & 24 & M & Full & SCN2A & NM_021007.3:c.4480C >A p.(Gln1494Lys) ${ }^{\star}$ & LP & Monoallelic; de novo \\
\hline
\end{tabular}




\begin{tabular}{|c|c|c|c|c|c|c|c|}
\hline Family & $\begin{array}{l}\text { Age, } \\
\text { years }\end{array}$ & Sex & Contribution & Gene & Variants & ACMG & Inheritance \\
\hline 90 & 49 & $\mathrm{~F}$ & Full & SHOC2 & NM_007373.4:C.519G >A p.(Met173lle) & LP & Monoallelic; unknown \\
\hline 91 & 56 & M & Partial & SLC2OA2 & NM_001257180.2:c.852delC p.(Ile1285fs)* & LP & Monoallelic; unknown \\
\hline \multirow[t]{2}{*}{92} & \multirow[t]{2}{*}{25} & \multirow[t]{2}{*}{ M } & \multirow[t]{2}{*}{ Full } & \multirow[t]{2}{*}{$S L C 52 A 2$} & NM_001363118.2:c.368T >C p.(Leu123Pro) & $P$ & \multirow[t]{2}{*}{ Biallelic } \\
\hline & & & & & NM_001363118.2:c.916G >A p.(Gly306Arg) & $P$ & \\
\hline 93 & 11 & $\mathrm{~F}$ & Full & SOS1 & NM_005633.3.3:c.1294T >C p.(Trp432Arg) & $P$ & Monoallelic; de novo \\
\hline 94 & 12 & M & Partial & TAB2 & NM_001292034.3: C.-90+1G >C & LP & Monoallelic; de novo \\
\hline 95 & 7 & $\mathrm{~F}$ & Full & TANGO2 & Deletion exons 3-9; homozygous & $P$ & Biallelic \\
\hline 96 & 58 & M & Partial & $T T \mathrm{~N}$ & NM_001267550.2:C.59926+1G >A & $P$ & Monoallelic unknown \\
\hline 97 & 73 & M & Full & TTR & NM_000371.3:c.407A >C p.(Tyr136Ser) & $\mathrm{P}$ & Monoallelic unknown \\
\hline 98 & 22 & $\mathrm{~F}$ & Full & ZBTB20 & NM_001164342.2: c.1916G >A p.(Cys639Tyr)* & LP & Monoallelic unknown \\
\hline \multicolumn{8}{|c|}{ Probable } \\
\hline 99 & 59 & $\mathrm{~F}$ & Full & CTNNB1 & NM_001904.4:c.2315delA p.(Asn772fs)* & LP & Monoallelic; unknown \\
\hline \multirow[t]{2}{*}{100} & \multirow[t]{2}{*}{0} & \multirow[t]{2}{*}{$M$} & \multirow[t]{2}{*}{ Full } & \multirow[t]{2}{*}{$M Y B P C 3$} & NM_000256.3:c.1357_158delCCp.(Pro453fs) & $\mathrm{P}$ & \multirow{2}{*}{ Biallelic } \\
\hline & & & & & NM_000256.3:c.1576G >C p.(Ala526Pro)* & LP & \\
\hline 101 & 22 & $\mathrm{~F}$ & Full & PEX16 & NM_057174.2:c.851A >C p.(Tyr284Ser)*; homozygous & VUS & Biallelic \\
\hline \multicolumn{8}{|c|}{ Possible } \\
\hline 102 & 60 & $\mathrm{~F}$ & Full & MARS1 & NM_004990.4:c.493_495delGAG p.(Glu165del)* & LP & Monoallelic; unknown \\
\hline 103 & 52 & M & Full & MYH2 & NM_017534.6:c.2387C >A p.(Ala796Asp)* & VUS & Monoallelic; unknown \\
\hline \multirow[t]{2}{*}{104} & \multirow[t]{2}{*}{13} & \multirow[t]{2}{*}{ M } & \multirow[t]{2}{*}{ Full } & \multirow[t]{2}{*}{ MYO9A } & NM_006901.4:c.6796A >T p.(Asn2266Tyr)* & VUS & \multirow[t]{2}{*}{ Biallelic } \\
\hline & & & & & NM_006901.4:c.1574A >T p.(Glu525Val)* & VUS & \\
\hline \multicolumn{8}{|c|}{$\begin{array}{l}\text { Genetic diagnoses made in } 65 \text { families, including age and sex of participants, contribution to phenotype from this gene (full or partial), gene, variants, American College of Medical Genetics } \\
\text { (ACMG) variant classification ( } P=\text { pathogenic, } L P=\text { likely pathogenic, VUS=variant of uncertain clinical significance), and inheritance pattern. } \\
\text { For family } 84 \text {, full phenotype is explained by two genetic diagnoses. In family } 77, \text { NPHP1 homozygous deletion explains phenotype in sibling with severe phenotype (with childhood onset end } \\
\text { stage renal disease) and is heterozygous in other sibling. } \\
\text { AD=autosomal dominant; } F=\text { female; } M=\text { male; XLR=X linked recessive. } \\
\text { * Novel variants } \\
\text { tPreviously published families. }\end{array}$} \\
\hline
\end{tabular}

older people owing to the financial cost and perceived lack of immediate management implications. Patients in the 30-39 years age group were the least likely to be recruited and had a low diagnostic rate. A common reason for seeking a referral to clinical genetics is for reproductive advice, so we speculate that people in this age group are more likely to have been reviewed by a clinical geneticist and offered up to date genetic testing (such as panel testing) in a clinical setting, meaning that fewer patients did not have a diagnosis and the remaining ones were more difficult to diagnose.

The relatively high frequency of partial diagnoses in this study (4\%) may reflect our current knowledge of the phenotypic spectrum of ultra-rare genetic diseases,

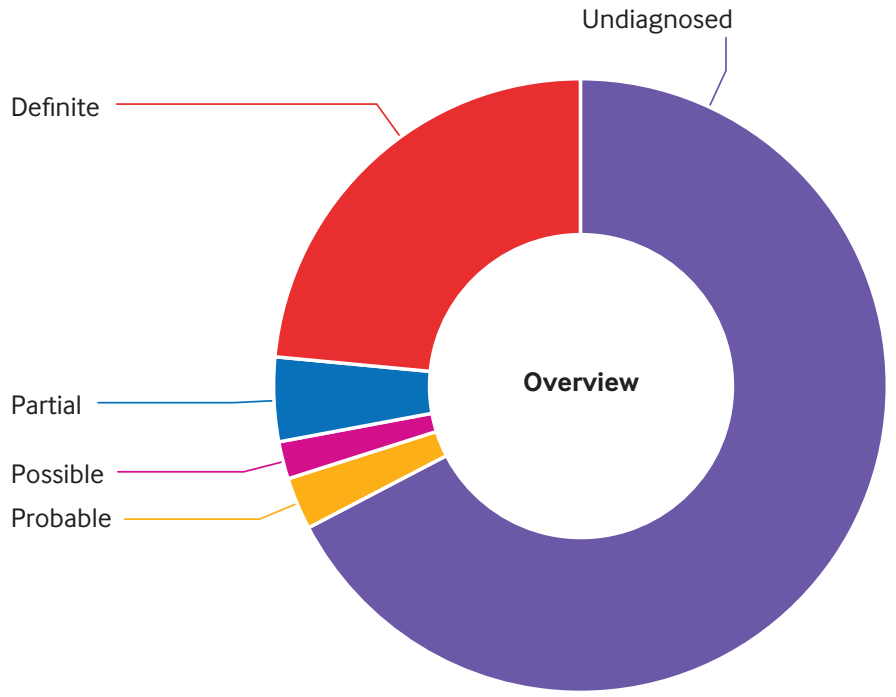

Fig 4 | Overview of proportion of families with and without diagnosis and some of the features we have not ascribed to the causal variants may actually be due to the underlying mutations. This is likely to be a particular problem for mitochondrial disorders because of their diverse phenotypes, some of which are only just being recognised, ${ }^{36}$ but will become easier as our knowledge base increases.

\section{Strengths and weaknesses of study in relation to other studies}

Our study had several strengths, including the large sample size and the fact that patients were recruited nationally from both secondary and tertiary care, meaning that the findings have a wider relevance than those of studies focused on highly selected groups identified by specialist centres. In addition, the use of HPO terms allowed us to analyse phenotype data in a systematic way, and we contacted clinicians for detailed phenotypic information in selected participants. Our analysis of the nuclear genes included copy number variants and short tandem repeat expansions in addition to single nucleotide variants and small insertion-deletion variants, and we also studied mtDNA variants with a heteroplasmy level $>1 \%$. The advantage of using whole genome sequencing rather than whole exome sequencing is that it is easier to pick up copy number variations, repeat expansions, and lower level heteroplasmies. Weaknesses are that we have not explored novel disease genes or variants in the non-coding regions, other than previously published variants, and tracing all family members was not possible.

The previously published study using whole genome sequencing in patients with suspected mitochondrial 


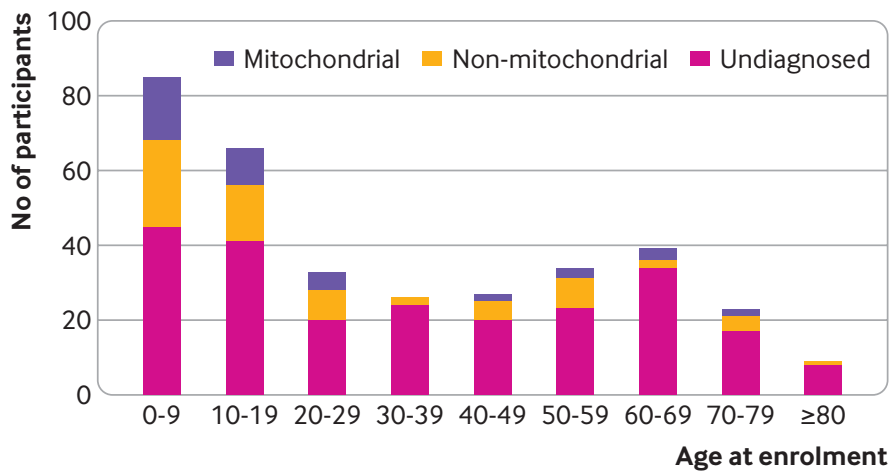

Fig 5 | Age distribution of participants at time of enrolment and type of diagnoses made. Diagnostic yield was higher in younger participants, but diagnoses were still being made in patients enrolled in their 70 s and $80 \mathrm{~s}$

disease looked at a cohort of 40 children recruited from four paediatric genetic metabolic centres in Australia. ${ }^{19}$ Of these, 34/40 had a probable or definite mitochondrial disorder according to the modified Nijmegen Mitochondrial Disease Criteria score (between 5 and 12) and 28 had abnormal respiratory chain enzyme activities. Analysis of nuclear and mtDNA enabled a definitive genetic diagnosis for $55 \%$ of patients, and a likely molecular diagnosis in 67\%, with $18 \%$ having a non-mitochondrial disorder. The higher diagnostic yield in this study likely reflects the section criteria, which focused on children within trios identified by national specialist clinics, ${ }^{19}$ compared with our more inclusive recruitment criteria whereby around two thirds of our probands were adults and only $46 \%$ of families were recruited as trios.

Several studies using whole exome sequencing in patients with suspected mitochondrial disorders have been reviewed recently. ${ }^{46}$ Most of these studies were conducted in highly selected patients seen in specialist centres, ${ }^{101216}$ focusing on childhood onset disorders

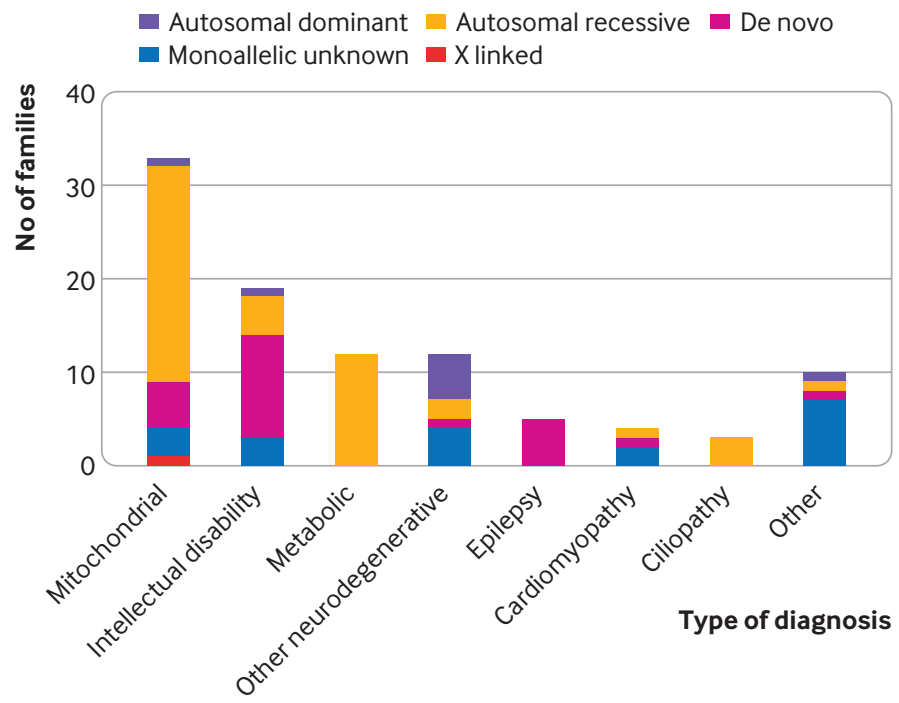

Fig 6 | Types of nuclear genetic disorder identified. Inheritance patterns in nuclear mitochondrial disorders and different types of non-mitochondrial disorders. Most families with nuclear mitochondrial disorders showed autosomal recessive inheritance. De novo dominant pathogenic variants were common in families with developmental disorders causing intellectual disability and in epileptic encephalopathies (apart from Wortmann and colleagues' study, ${ }^{14}$ which included patients up to 27 years of age). Their stringent recruitment criteria led to higher diagnostic yields (57-68\%) and lower numbers of non-mitochondrial diagnoses compared with our study. Previous exome studies of larger cohorts have also tended to have a lower diagnostic yield (35-39\%), ${ }^{1415}$ which is thought to be more reflective of everyday clinical practice. ${ }^{46}$ Non-mitochondrial disorders have been found in some of the previous studies, but in much smaller numbers than in our study. The recruitment criteria in our study were broad, which is reflective of mainstreaming of genomic medicine, and we studied both adults and children. Most participants (64\%) did not have muscle biopsy results available at the time of referral, whereas most participants in previous studies had evidence of mitochondrial dysfunction. The more inclusive eligibility criteria have led to us finding a wide range of different genetic diagnoses. The finding that a large number of patients had non-mitochondrial disorders is important because these diagnoses would have been missed if the participants had been investigated only for mitochondrial disorders through muscle biopsy, a specific mitochondrial gene panel, or both. This would have led to missed opportunities for treatment, surveillance, and reproductive management. Our findings highlight the difficulty of diagnosing these rare multisystem disorders clinically and the need to keep an open mind about the differential diagnosis.

Considering other studies of integration of whole genome sequencing into healthcare, Stranneheim and colleagues describe the results for 3219 rare disease patients recruited in Stockholm, Sweden, between 2015 and 2019. ${ }^{47}$ Their study involved much more direct collaboration between academia, healthcare, and their SciLifeLab (which provides whole genome sequencing), which were all located in the same city. This meant that potential diagnoses were discussed and specialist advice was fed back quickly into clinical practice. Specialist clinicians could also access and analyse the data together with clinical scientists. The 100000 Genomes Project used a different model with the bioinformatics managed centrally and the clinical interpretation of variants done by genomic medicine centre laboratories. Researchers were able to access pseudonymised data. The limited ability of researchers and clinicians to discuss potential diagnoses together and the long turnaround time were disadvantages compared with the Swedish model.

\section{Unanswered questions and future research}

The participants in this study had standard of care NHS genetic testing before enrolment. The use of whole genome sequencing as a first genetic test for suspected mitochondrial disorders has not been directly explored. The only mitochondrial diseases that cannot be diagnosed by whole genome sequencing of DNA extracted from blood are extremely rare muscle specific mtDNA mutations and large mtDNA deletions, which usually have a very specific clinical phenotype. On the basis of known epidemiology, ${ }^{148}$ these muscle 


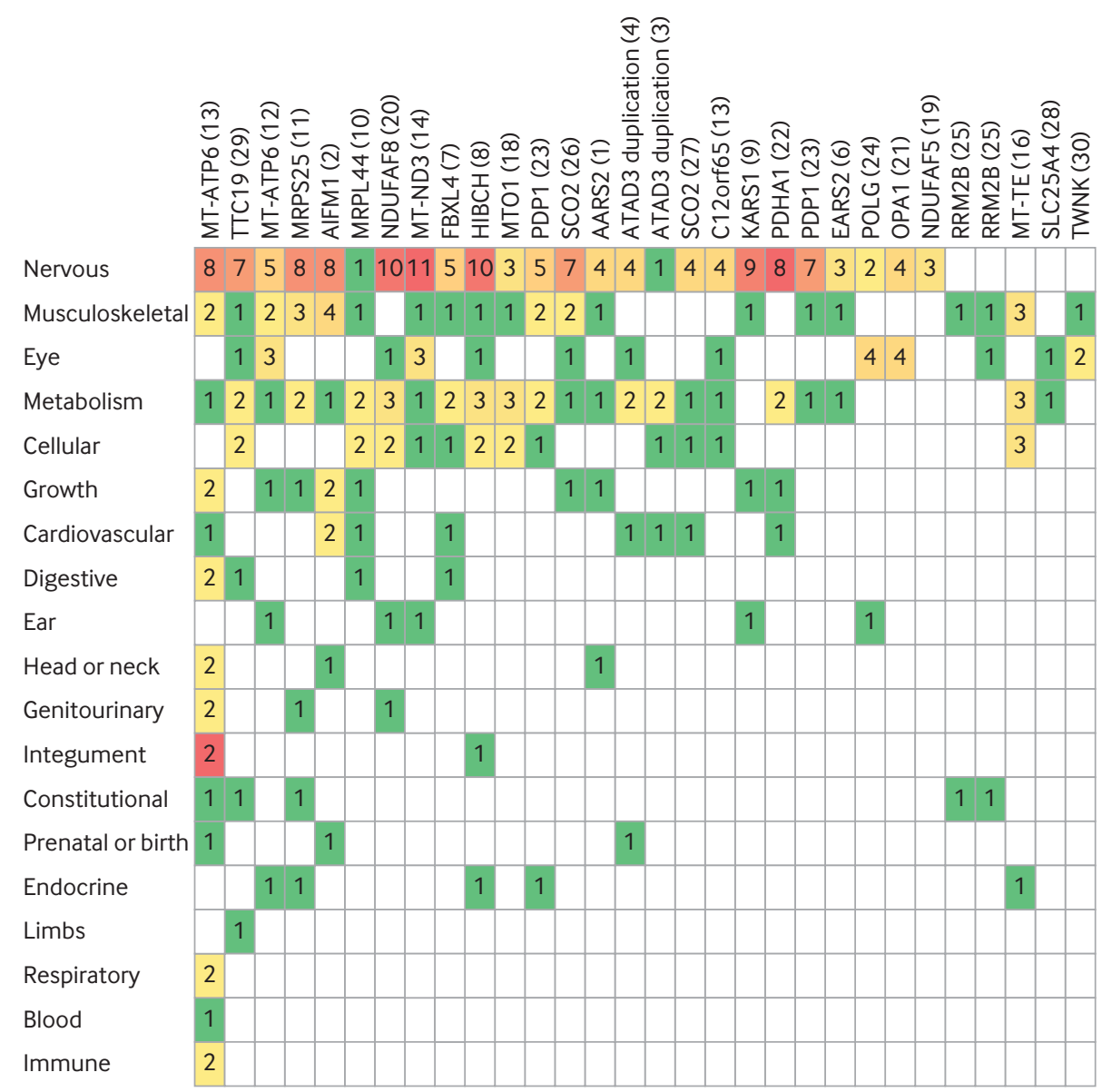

Fig 7 | Human Phenotype Ontology (HPO) terms in patients with mitochondrial diagnoses. HPO ancestor systems for HPO terms recorded in participants with definite mitochondrial diagnoses (excluding partial diagnoses). Each column represents one participant (family number in brackets). Each row represents a different HPO ancestor system, listed in order of how frequently they were affected, with nervous system at top. Numbers indicate how many of participant's HPO terms related to HPO ancestor system (eg, nervous system or musculoskeletal system). Colours go from green through to red as number of terms related to the HPO ancestor system increases

specific mtDNA mutations and deletions account for approximately $11.5 \%$ of patients with a genetically confirmed mitochondrial disorder. Therefore, mitochondrial disorders can be diagnosed by whole genome sequencing of DNA extracted from blood in nearly $90 \%$ of patients. ${ }^{49}$ Additionally, whole genome sequencing can diagnose other monogenic disorders that have similar clinical features, so we would expect a very high diagnostic yield. Finally, by carrying out this study we have identified a cohort of patients with suspected mitochondrial disease in whom the likely diagnosis is contained within the whole genome sequencing data but cannot be distinguished from background sequence variation. This will form a useful resource for the discovery of future mitochondrial disease genes that are not in the coding space and thus are not detectable by whole exome sequencing. Large scale mtDNA rearrangements are not reliably detected in DNA extracted from blood in adults, meaning that patients with suspected progressive external ophthalmoplegia and Kearns-Sayre syndrome are investigated by muscle biopsy. ${ }^{20}$ Future research will determine whether deep mtDNA sequencing will detect these large scale mtDNA rearrangements in DNA extracted from blood or another accessible tissue such as urinary epithelium.

\section{Policy implications}

Our findings indicate that whole genome sequencing is a useful diagnostic test in patients with suspected mitochondrial disorders recruited from secondary and tertiary care settings. We recommend that whole genome sequencing should be offered early in the diagnostic pathway in a patient's local secondary or tertiary care centre and before invasive tests such as a muscle biopsy. Exceptions to this would be patients whose clinical features are highly suggestive of a specific cause that can be confirmed by a single gene test or common mtDNA mutation testing, as well as patients with progressive external ophthalmoplegia, which is diagnosed in most patients by testing mtDNA from a muscle biopsy sample for large scale rearrangements. Referral to a specialised mitochondrial clinic should be considered if whole genome sequencing is uninformative. Further investigations likely to increase the diagnostic yield beyond whole genome sequencing include laboratory studies of mitochondrial function and other "omics" 


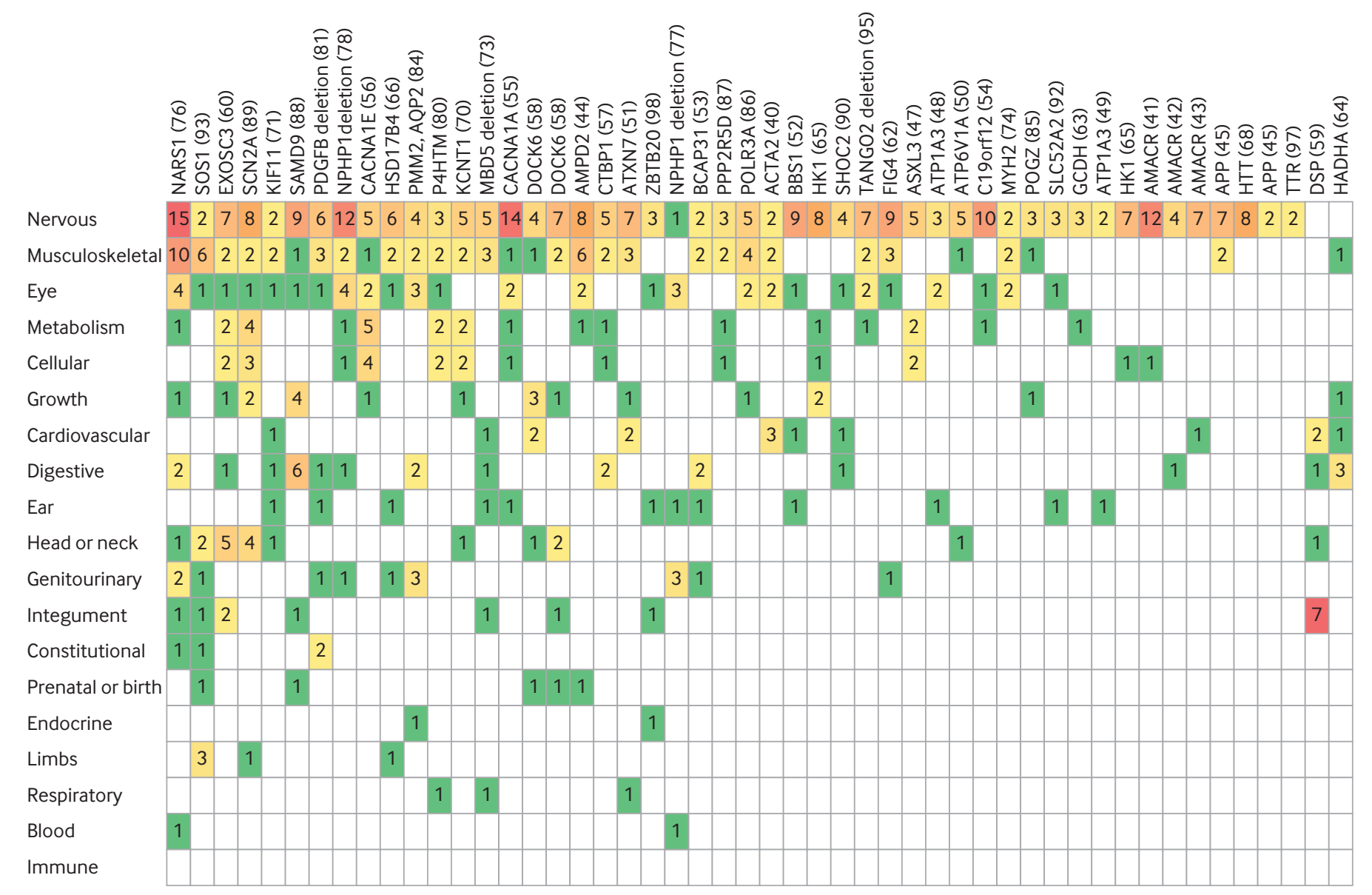

Fig 8 | Comparison of Human Phenotype Ontology (HPO) terms in patients with non-mitochondrial diagnoses. HPO ancestor systems for HPO terms recorded in participants with definite non-mitochondrial diagnoses (excluding partial diagnoses). Each column represents one participant (family number in brackets). Each row represents a different HPO ancestor system, listed in order of how frequently they were affected, with nervous system at top. Numbers indicate how many of participant's HPO terms related to HPO ancestor system (e.g., nervous system or musculoskeletal system). Colours go from green through to red as number of terms related to the HPO ancestor system increases

approaches including transcriptomics, which provided an additional diagnosis in $10 \%$ of patients with suspected mitochondrial disease in one study, ${ }^{50}$ proteomics, and metabolomics.

The integration of whole genome sequencing into healthcare also has wider policy implications. The relatively high number of patients with probable or

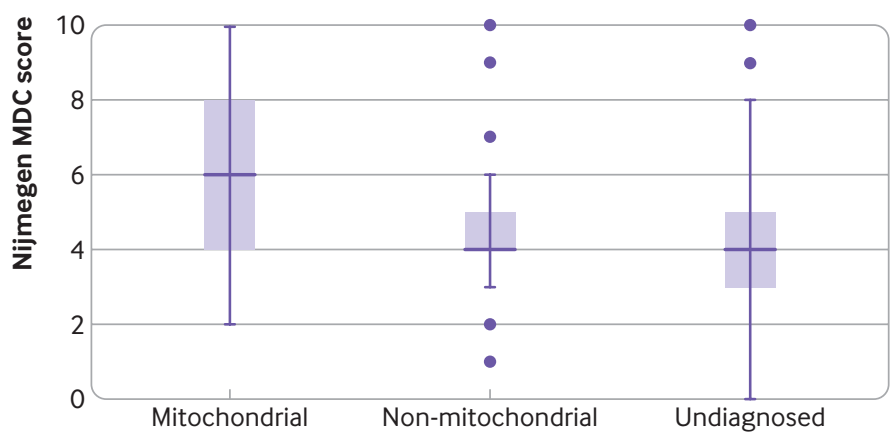

Fig 9 | Human Phenotype Ontology modified Nijmegen Mitochondrial Diagnostic Criteria (MDC) scores in participants with confirmed genetic diagnoses of mitochondrial and non-mitochondrial disorders and in participants with no diagnosis. Participants with "probable," "possible," and "partial" diagnoses were excluded from this analysis. MDC scores were higher in patients with mitochondrial diagnoses than non-mitochondrial diagnoses or no diagnosis $(P<0.05)$ possible diagnoses partly reflects the lack of capacity for the functional evaluation of variants of uncertain clinical significance-for example, through splicing assays or Western blotting. Resources should also be made available for the regular reanalysis of whole genome sequencing data, either at specified time intervals in patients without a diagnosis or on a clinician's request. The reanalysis of exome data has been shown to significantly increase diagnostic yield, mainly owing to newly discovered disease genes. ${ }^{51}$ Finally, rapid trio whole genome sequencing should be offered in acutely unwell individuals with suspected mitochondrial disorders, ${ }^{17}$ so that the results can help to guide clinical management. In the UK, rapid trio exome sequencing is available only for acutely unwell children.

\section{AUTHOR AFFILIATIONS}

${ }^{1}$ Department of Clinical Neurosciences, School of Clinical Medicine, University of Cambridge, Cambridge, UK

${ }^{2}$ Medical Research Council Mitochondrial Biology Unit, University of Cambridge, Cambridge, UK

${ }^{3}$ East Anglian Medical Genetics Service, Cambridge University Hospitals NHS Foundation Trust, Cambridge, UK

${ }^{4}$ William Harvey Research Institute, Queen Mary University of London, London, UK

${ }^{5}$ Department of Paediatrics, University of Cambridge, Cambridge, UK 
${ }^{6}$ Department of Neuromuscular Diseases, UCL Queen Square Institute of Neurology and The National Hospital for Neurology and Neurosurgery, London, UK

${ }^{7}$ Department of Clinical Genetics and Genomic Medicine, Great Ormond Street Hospital for Children NHS Foundation Trust, London, UK ${ }^{8}$ NHS North Thames Genomic Laboratory Hub, Great Ormond Street Hospital for Children NHS Foundation Trust, London, UK

${ }^{9}$ Translational and Clinical Research Institute, Faculty of Medical Sciences, Newcastle University, Newcastle upon Tyne, UK

${ }^{10}$ Northern Genetics Service, Newcastle Hospitals NHS Foundation Trust, International Centre for Life, Newcastle upon Tyne, UK

${ }^{11}$ Department of Clinical Genetics, Guy's and St Thomas' NHS Foundation Trust, London, UK

${ }^{12} \mathrm{NHS}$ Highly Specialised Services for Rare Mitochondrial Disorders - Oxford Centre, Oxford University Hospitals NHS Foundation Trust, Oxford, UK

${ }^{13}$ Central and South Genome Medicine Service Alliance and Genomics Laboratory Hub, Birmingham Women's and Children's NHS Foundation Trust, Birmingham, UK

${ }^{14}$ Institute of Cancer and Genomic Sciences, College of Medical and Dental Sciences, University of Birmingham, Birmingham, UK

${ }^{15}$ Manchester Centre for Genomic Medicine, St Mary's Hospital, Manchester, UK

${ }^{16}$ Department of Clinical Genetics, University Hospitals Bristol, Bristol, UK

${ }^{17}$ Department of Brain Sciences, London Institute of Medical Sciences, Mansfield Centre for Inovation, Imperial College, Hammersmith Hospital, London, UK

${ }^{18}$ Liverpool Centre for Genomic Medicine, Liverpool Women's Hospital, Liverpool, UK

${ }^{19}$ Genomics England, William Harvey Research Institute, Queen Mary University of London, London, UK

${ }^{20}$ Neurology and Mitochondrial Disorders Genomics Clinical Interpretation Partnership, William Harvey Research Institute, Queen Mary University of London, London, UK

${ }^{21}$ Metabolic Unit, Great Ormond Street Hospital for Children NHS Foundation Trust, London, UK

${ }^{22}$ Mitochondrial Research Group, Department of Genetics and Genomic Medicine, UCL Great Ormond Street Institute of Child Health, London, UK

We thank the National Institute for Health Research Wellcome Clinical Research Facility, Birmingham, for recruiting West Midlands participants.

Members of the Genomics England Research Consortium: I C Ambrose, P Arumugam, R Bevers, M Bleda, F Boardman-Pretty, C R Boustred, H Brittain, M I Caulfield, G C Chan, G Elgar, T Fowler, A Giess, A Hamblin, S Henderson, T J P Hubbard, R Jackson, LI Jones, D Kasperaviciute, M Kayikci, A Kousathanas, L Lahnstein, S E A Leigh, I U S Leong, F J Lopez, F Maleady-Crowe, M McEntegart, F Minneci, L Moutsianas, M Mueller, N Murugaesu, A C Need, P O'Donovan, C A Odhams, C Patch, M B Pereira, D Perez-Gil, J Pullinger, T Rahim, A Rendon, T Rogers, K Savage, K Sawant, R H Scott, A Siddiq, A Sieghart, S C Smith, A Sosinsky, A Stuckey, M Tanguy, A L Taylor Tavares, E R A Thomas, S R Thompson, A Tucci, M J Welland, E Williams, K A Witkowska, S M Wood.

Contributors: KRS, RH, and PFC designed the study. PFC and RH supervised the study. WW, CC, AT, and KA did the bioinformatic analysis. KRS, RH, AT, RDSP, RQ, EB, MH, EC, JS, PB, DJ, LI, CN, TB, AS, CD, SFS, RF, HH, SR, and PFC recruited patients. KRS, RH, AT, TR EA, CF, DM, and PFC did clinical interpretation of genetic variants. MC was the chief scientific officer for Genomics England and oversaw the 100000 Genomes Project. KRS and PFC wrote the manuscript. All authors reviewed and revised the manuscript. PFC is the guarantor. The corresponding author attests that all listed authors meet authorship criteria and that no others meeting the criteria have been omitted.

Funding: This research was made possible through access to the data and findings generated by the 100000 Genomes Project. The 100000 Genomes Project is managed by Genomics England Ltd (a wholly owned company of the Department of Health and Social Care). The 100000 Genomes Project is funded by the National Institute for Health Research (NIHR) and NHS England. The Wellcome Trust, Cancer Research UK, and the Medical Research Council (MRC) have also funded research infrastructure. The 100000 Genomes Project uses data provided by patients and collected by the National Health
Service as part of their care and support. PFC is a Wellcome Trust principal research fellow (212219/Z/18/Z), and a UK NIHR senior investigator, who receives support from the MRC Mitochondrial Biology Unit (MC_UU_00015/9), the MRC International Centre for Genomic Medicine in Neuromuscular Disease (MR/S005021/1), the Leverhulme Trust (RPG-2018-408), an MRC research grant (MR/ S035699/1), an Alzheimer's Society project grant (AS-PG-18b-022), and the NIHR Biomedical Research Centre based at Cambridge University Hospitals NHS Foundation Trust and the University of Cambridge. KRS is supported by Addenbrooke's Charitable Trust and the MRC International Centre for Genomic Medicine in Neuromuscular Disease (MR/S005021/1). RH is a Wellcome Trust investigator (109915/Z/15/Z), who receives support from the MRC (UK) (MR/ N025431/1), the European Research Council, the Newton Fund (UK/ Turkey, MR/N027302/1), the Evelyn Trust, and the Lily Foundation. The University College London Hospitals/University College London Queen Square Institute of Neurology sequencing facility receives a proportion of funding from the Department of Health's NIHR biomedical research centres funding scheme. The clinical and diagnostic "Rare Mitochondrial Disorders" Service in London is funded by the UK NHS Highly Specialised Commissioners. RDSP is supported by an MRC clinician scientist fellowship (MR/S002065/1). RDSP and MGH are funded by an MRC strategic award to establish an International Centre for Genomic Medicine in Neuromuscular Diseases (ICGNMD) (MR/S005021/1). RF is funded by Imperial NIHR BRC Imperial College Healthcare Trust. All research at Great Ormond Street Hospital NHS Foundation Trust and UCL Great Ormond Street Institute of Child Health is made possible by the NIHR Great Ormond Street Hospital Biomedical Research Centre. The views expressed are those of the author(s) and not necessarily those of the NHS, the NIHR, or the Department of Health and Social Care.

Competing Interests: All authors have completed the ICMJE uniform disclosure form at www.icmje.org/disclosure-of-interest/ and declare: support for the submitted work as detailed above; no financial relationships with any organisations that might have an interest in the submitted work in the previous three years; no other relationships or activities that could appear to have influenced the submitted work.

Ethical approval: Ethical approval for the 100000 Genomes Project was received from the HRA Committee East of England Cambridge South (REC Ref 14/EE/1112). All participants provided written informed consent.

Data sharing: 100000 Genomes Project data are available to researchers and clinicians through joining a Genomics England Clinical Interpretation Partnership) (www.genomicsengland.co.uk/ about-gecip/joining-research-community/)

The lead author (the manuscript's guarantor) affirms that the manuscript is an honest, accurate, and transparent account of the study being reported; that no important aspects of the study have been omitted; and that any discrepancies from the study as planned (and, if relevant, registered) have been explained.

Dissemination to participants and related patient and public communities: We will distribute the article to clinicians and advocacy groups including the Lily Foundation, Muscular Dystrophy UK, and the United Mitochondrial Disease Foundation. We will distribute findings on social media and a plain language summary on the Cambridge Clinical Mitochondrial Research Group website (https://wwwneurosciences.medschl.cam.ac.uk/mitocamb/). We will issue a press release through the University of Cambridge and Genomics England.

Provenance and peer review: Not commissioned; externally peer reviewed.

This is an Open Access article distributed in accordance with the terms of the Creative Commons Attribution (CC BY 4.0) license, which permits others to distribute, remix, adapt and build upon this work, for commercial use, provided the original work is properly cited. See: http://creativecommons.org/licenses/by/4.0/.

1 Gorman GS, Schaefer AM, Ng Y, et al. Prevalence of nuclear and mitochondrial DNA mutations related to adult mitochondrial disease. Ann Neurol 2015.77.753-9. doi:10.1002/ana.24362

2 Vafai SB, Mootha VK. Medicine. A common pathway for a rare disease?Science 2013:342:1453-4. doi:10.1126/science.1248449 Jurkute N, Yu-Wai-Man P. Leber hereditary optic neuropathy: bridging the translational gap. Curr Opin Ophthalmol 2017;28:403-9. doi:10.1097/ICU.0000000000000410

4 Gorman GS, Chinnery PF, DiMauro S, et al. Mitochondrial diseases. Nat Rev Dis Primers 2016;2:16080. doi:10.1038/nrdp.2016.80

5 Rahman J, Rahman S. Mitochondrial medicine in the omics era. Lancet 2018:391:2560-74 doi:10 1016/50140-6736(18)30727-X 
6 Rahman J, Noronha A, Thiele I, Rahman S. Leigh map: A novel computational diagnostic resource for mitochondrial disease. Ann Neurol 2017;81:9-16. doi:10.1002/ana.24835

7 Black N, Martineau F, Manacorda T. Diagnostic odyssey for rare diseases: exploration of potential indicators. 2015 https://piru.ac.uk/ assets/files/Rare\%20diseases\%20Final\%20report.pdf.

8 Grier J, Hirano M, Karaa A, Shepard E, Thompson JLP. Diagnostic odyssey of patients with mitochondrial disease: Results of a survey. Neurol Genet 2018:4:e230. doi:10.1212 NXG.0000000000000230

9 McFarland R, Taylor RW, Turnbull DM. A neurological perspective on mitochondrial disease. Lancet Neurol 2010;9:829-40. doi:10.1016/ S1474-4422(10)70116-2

10 Taylor RW, Pyle A, Griffin H, et al. Use of whole-exome sequencing to determine the genetic basis of multiple mitochondrial respiratory chain complex deficiencies. JAMA 2014;312:68-77. doi:10.1001/ jama.2014.7184

11 Pronicka E, Piekutowska-Abramczuk D, Ciara E, et al. New perspective in diagnostics of mitochondrial disorders: two years' experience with whole-exome sequencing at a national paediatric centre. J Transl Med 2016;14:174. doi:10.1186/s12967-016-0930-9

12 Puusepp S, Reinson K, Pajusalu S, et al. Effectiveness of whole exome sequencing in unsolved patients with a clinical suspicion of a mitochondrial disorder in Estonia. Mol Genet Metab Rep 2018;15:80-9. doi:10.1016/i.ymgmr.2018.03.004

13 Lieber DS, Calvo SE, Shanahan K, et al. Targeted exome sequencing of suspected mitochondrial disorders. Neurology 2013;80:1762-70. doi:10.1212/WNL.0b013e3182918c40

14 Wortmann SB, Koolen DA, Smeitink JA, van den Heuvel L, Rodenburg RJ. Whole exome sequencing of suspected mitochondrial patients in clinical practice. J Inherit Metab Dis 2015;38:437-43. doi:10.1007/ s10545-015-9823-y

15 Kohda M, Tokuzawa Y, Kishita Y, et al. A Comprehensive Genomic Analysis Reveals the Genetic Landscape of Mitochondrial Respiratory Chain Complex Deficiencies. PLoS Genet 2016;12:e1005679. doi:10.1371/journal.pgen.1005679

16 Theunissen TEJ, Nguyen M, Kamps R, et al. Whole Exome Sequencing Is the Preferred Strategy to Identify the Genetic Defect in Patients With a Probable or Possible Mitochondrial Cause. Front Genet 2018;9:400. doi:10.3389/fgene.2018.00400

17 French CE, Delon I, Dolling H, et al, NIHR BioResource-Rare Disease Next Generation Children Project. Whole genome sequencing reveals that genetic conditions are frequent in intensively ill children. Intensive Care Med 2019;45:627-36. doi:10.1007/s00134-019 05552-x

18 Poulton J, Steffann J, Burgstaller J, McFarland R, workshop participants. 243rd ENMC international workshop: Developing guidelines for management of reproductive options for families with maternally inherited mtDNA disease, Amsterdam, the Netherlands, 22-24 March 2019. Neuromuscul Disord 2019;29:725-33. doi:10.1016/j.nmd.2019.08.004

19 Riley LG, Cowley MJ, Gayevskiy V, et al. The diagnostic utility of genome sequencing in a pediatric cohort with suspected mitochondrial disease. Genet Med 2020;22:1254-61. doi:10.1038/ s41436-020-0793-6

20 Schon KR, Ratnaike T, van den Ameele J, Horvath R, Chinnery PF. Mitochondrial Diseases: A Diagnostic Revolution. Trends Genet 2020;36:702-17. doi:10.1016/j.tig.2020.06.009

21 Turnbull C, Scott RH, Thomas E, et al, 100000 Genomes Project. The 100000 Genomes Project: bringing whole genome sequencing to the NHS. BMJ 2018;361:k1687. doi:10.1136/bmj.k1687

22 Turro E, Astle WJ, Megy K, et al, NIHR BioResource for the 100,000 Genomes Project. Whole-genome sequencing of patients with rare diseases in a national health system. Nature 2020;583:96-102. doi:10.1038/s41586-020-2434-2

23 Köhler S, Doelken SC, Mungall Cl, et al. The Human Phenotype Ontology project: linking molecular biology and disease through phenotype data. Nucleic Acids Res 2014;42:D966-74. doi:10.1093/ nar/gkt1026

24 Bernier FP, Boneh A, Dennett X, Chow CW, Cleary MA, Thorburn DR. Diagnostic criteria for respiratory chain disorders in adults and children. Neurology 2002;59:1406-11. doi:10.1212/01. WNL 0000033795.17156 .00

25 Morava E, van den Heuvel L, Hol F, et al. Mitochondrial disease criteria: diagnostic applications in children. Neurology 2006;67:1823-6. doi:10.1212/01.wnl.0000244435.27645.54

26 Caulfield M, Davies J, Dennys M, Elbahy L, Fowler T. The National Genomics Research and Healthcare Knowledgebase: Amendment to the 100,000 Genomes Project Protocol v4. 2019. https://www. genomicsengland.co.uk/wp-content/uploads/2019/08/The-NationalGenomics-Research-and-Healthcare-Knowledgebase-v5-1.pdf.

27 Wei W, Tuna S, Keogh MJ, et al, NIHR BioResource-Rare Diseases, 100,000 Genomes Project-Rare Diseases Pilot. Germline selection shapes human mitochondrial DNA diversity. Science 2019;364:eaau6520. doi:10.1126/science.aau6520
28 Rimmer A, Phan H, Mathieson I, et al, WGS500 Consortium. Integrating mapping-, assembly- and haplotype-based approaches for calling variants in clinical sequencing applications. Nat Genet 2014;46:912-8. doi:10.1038/ng.3036

29 Martin AR, Williams E, Foulger RE, et al. PanelApp crowdsources expert knowledge to establish consensus diagnostic gene panels. Nat Genet 2019;51:1560-5. doi:10.1038/s41588-019-0528-2

30 Baple E. Genomics England Rare Disease Results Guide. 2020. https:// www.genomicsengland.co.uk/about-genomics-england/the-100000genomes-project/information-for-gmc-staff/rare-disease-documents/.

31 Robinson PN, Köhler S, Oellrich A, et al, Sanger Mouse Genetics Project. Improved exome prioritization of disease genes through cross-species phenotype comparison. Genome Res 2014;24:340-8. doi:10.1101/gr.160325.113

32 Landrum MJ, Lee JM, Benson M, et al. ClinVar: improving access to variant interpretations and supporting evidence. Nucleic Acids Res 2018;46(D1):D1062-7. doi:10.1093/nar/gkx1153

33 Roller E, Ivakhno S, Lee S, Royce T, Tanner S. Canvas: versatile and scalable detection of copy number variants. Bioinformatics 2016:32:2375-7. doi:10.1093/bioinformatics/btw163

34 Ibanez K, Polke J, Hagelstrom T, et al. Whole genome sequencing for diagnosis of neurological repeat expansion disorders. bioRxiv 2020:2020.11.06.371716.

35 Calabrese C, Simone D, Diroma MA, et al. MToolBox: a highly automated pipeline for heteroplasmy annotation and prioritization analysis of human mitochondrial variants in high-throughput sequencing. Bioinformatics 2014;30:3115-7. doi:10.1093/bioinformatics/btu483

36 Ratnaike TE, Greene D, Wei W, et al. MitoPhen database: a human phenotype ontology-based approach to identify mitochondrial DNA diseases. Nucleic Acids Res 2021;49:9686-95. doi:10.1093/nar/ gkab726

37 Richards S, Aziz N, Bale S, et al, ACMG Laboratory Quality Assurance Committee. Standards and guidelines for the interpretation of sequence variants: a joint consensus recommendation of the American College of Medical Genetics and Genomics and the Association for Molecula Pathology. Genet Med 2015;17:405-24. doi:10.1038/gim.2015.30

38 Karczewski KJ, Francioli LC, Tiao G, et al, Genome Aggregation Database Consortium. The mutational constraint spectrum quantified from variation in 141,456 humans. Nature 2020;581:434-43. doi:10.1038/s41586-020-2308-7

39 Yates AD, Achuthan P, Akanni W, et al. Ensembl 2020. Nucleic Acids Res 2020;48(D1):D682-8.

40 Kopanos C, Tsiolkas V, Kouris A, et al. VarSome: the human genomic variant search engine. Bioinformatics 2019;35:1978-80. doi:10.1093/bioinformatics/bty897

41 Robinson JT, Thorvaldsdóttir H, Winckler W, et al. Integrative genomics viewer. Nat Biotechnol 2011;29:24-6. doi:10.1038/nbt.1754

42 Yung Tiet M, Lin Z, Gao F, Jennings MJ, Horvath R. Targeted Therapies for Leigh Syndrome: Systematic Review and Steps Towards a 'Treatabolome'. I Neuromuscul Dis 2021.

43 Tan TY, Lunke S, Chong B, et al. A head-to-head evaluation of the diagnostic efficacy and costs of trio versus singleton exome sequencing analysis. Eur J Hum Genet 2019;27:1791-9. doi:10.1038/s41431-019-0471-9

44 Wright CF, FitzPatrick DR, Firth HV. Paediatric genomics: diagnosing rare disease in children. Nat Rev Genet 2018;19:253-68. doi:10.1038/nrg.2017.116

45 Posey JE, Rosenfeld JA, James RA, et al. Molecular diagnostic experience of whole-exome sequencing in adult patients. Genet Med 2016:18:678-85. doi:10.1038/gim.2015.142

46 Stenton SL, Prokisch H. Genetics of mitochondrial diseases: Identifying mutations to help diagnosis. EBioMedicine 2020;56:102784. doi:10.1016/j.ebiom.2020.102784

47 Stranneheim H, Lagerstedt-Robinson K, Magnusson M, et al. Integration of whole genome sequencing into a healthcare setting: high diagnostic rates across multiple clinical entities in 3219 rare disease patients. Genome Med 2021;13:40. doi:10.1186/s13073021-00855-5

48 Castro-Gago M, Blanco-Barca MO, Campos-González Y, ArenasBarbero J, Pintos-Martínez E, Eirís-Puñal J. Epidemiology of pediatric mitochondrial respiratory chain disorders in northwest Spain. Pediatr Neurol 2006;34:204-11. doi:10.1016/j. pediatrneurol.2005.07.011

49 Raymond FL, Horvath R, Chinnery PF. First-line genomic diagnosis of mitochondrial disorders. Nat Rev Genet 2018;19:399-400. doi:10.1038/s41576-018-0022-1

50 Kremer LS, Bader DM, Mertes C, et al. Genetic diagnosis of Mendelian disorders via RNA sequencing. Nat Commun 2017;8:15824. doi:10.1038/ncomms15824

51 Liu P, Meng L, Normand EA, et al. Reanalysis of Clinical Exome Sequencing Data. N Engl J Med 2019;380:2478-80. doi:10.1056/NEJMc1812033

\footnotetext{
Web appendix: Supplementary materials Web appendix: Supplementary table G
} 